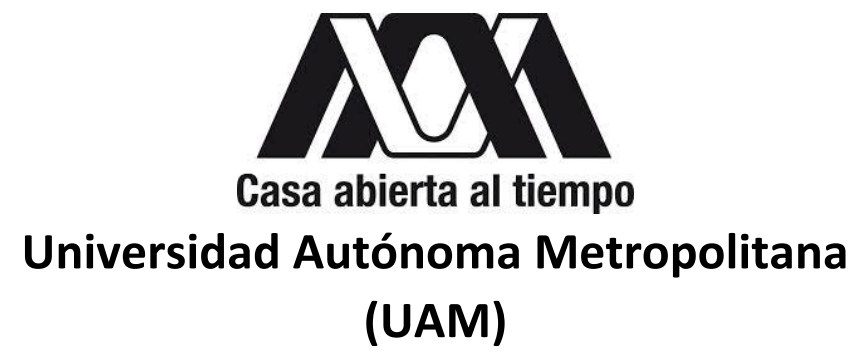

Maestría Integrado de Maestría y Doctorado en Ciencias Económicas (PIMDCE)

Evaluación de Reglas de Tasa de Interés de Política Monetaria: Un enfoque de Equilibrio General Estocástico Dinámico (DSGE)

Idónea de Comunicación de Resultados (ICR) para obtener el Título de Maestro en Ciencias Económicas

Presenta:

Boris Alfredo Luna Acevedo

Asesor:

Dr. Eddy Lizarazu Alanez 


\title{
Evaluación de Reglas de Tasa de Interés de Política Monetaria: Un enfoque de Equilibrio General Estocástico Dinámico (DSGE)
}

\begin{abstract}
Resumen
El presente documento de investigación realiza una evaluación de las reglas tasas de interés óptima expuesto en el documento de Clarida, Gali y Getler (1999), quienes consideran una familia de tasas de interés óptima bajo un enfoque de los Nuevos Keynesianos. Por tanto, el trabajo realiza una exposición de los modelos de equilibrio general y de las reglas de tasas de interés para luego evaluar mediante los instrumentos de calibración las respuestas ante shock de demanda, oferta y de tasa de interés. Los resultados muestran que bajo una regla de tasa de interés suavizada forward-looking las dos variables objetivas de política monetaria (producto e inflación) responden de manera moderada ante shock de demanda, oferta y de tasa de interés y que se disipa el efecto en promedio en menos de 2 o 3 trimestres.
\end{abstract}

Palabras Claves: Política Monetaria, tasa de interés, equilibrio general. 


\section{Contenido}

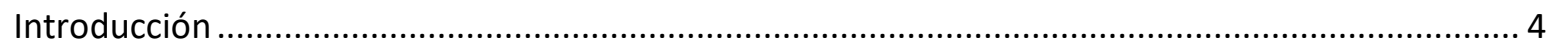

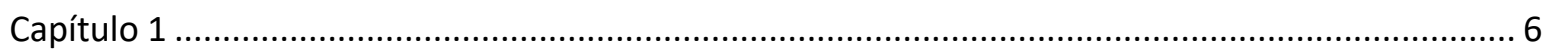

Elementos claves del Enfoque Nuevo Keynesiano y la elección de instrumento .............................. 6

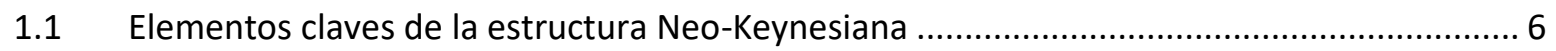

1.2 Reglas versus discrecionalidad y la elección de instrumentos............................................ 7

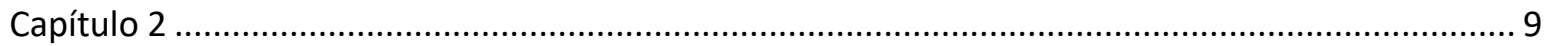

Fundamento Teórico de la Política Monetaria................................................................................ 9

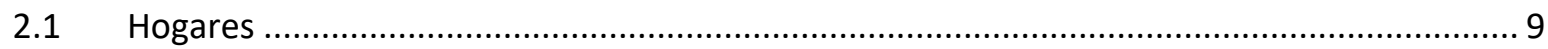

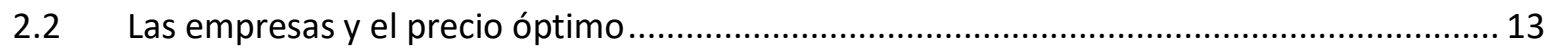

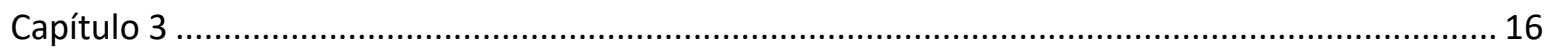

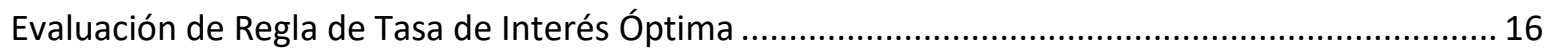

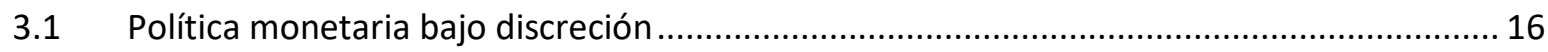

3.2 Compromiso de la Política Monetaria........................................................................... 19

3.2.1 Política monetaria bajo compromiso con restricciones................................................ 19

3.2.2 Política monetaria bajo compromiso sin restricciones ................................................ 21

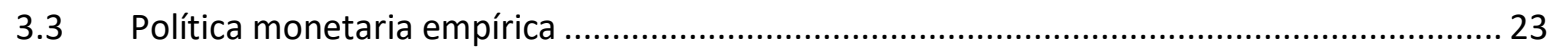

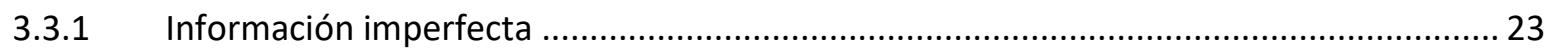

3.3.1.1 Pronóstico como objetivos intermedios ......................................................................... 24

3.3.1.2 Problema de elección de instrumentos: Tasa de interés versus agregados monetarios.. 25

3.3.1.3 Política conservadora: incertidumbre versus forward - looking ................................... 27

3.3.2 Reglas de tasa de interés bajo inflación endógena y producto persistente. ................... 28

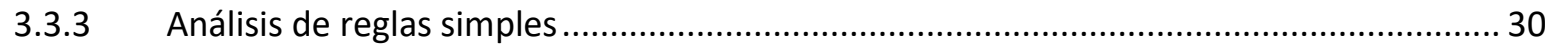

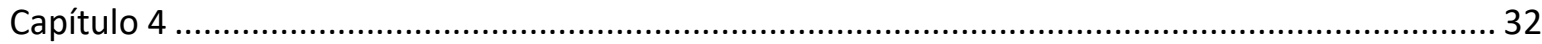

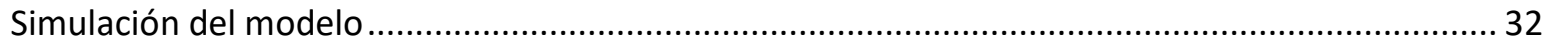

$4.1 \quad$ Resumen de las ecuaciones log-linealizada ....................................................................... 32

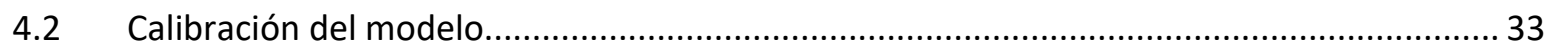

4.3 Análisis de Función Impulso Respuesta (FIR's) .................................................................... 34

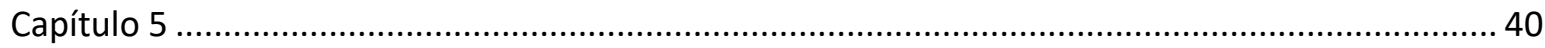

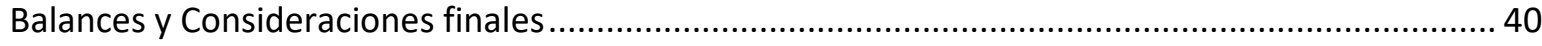

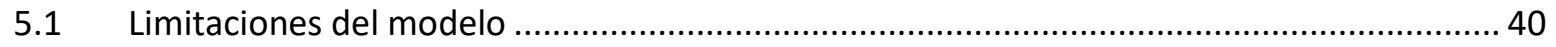

5.2 Conclusiones y perspectiva de la investigación .................................................................... 42 
Bibliografía

Anexo 45 


\section{Evaluación de Reglas de Tasa de Interés de Política Monetaria: Un enfoque de Equilibrio General Estocástico Dinámico (DSGE)}

\section{Introducción}

La macroeconomía tradicional basado en los simples modelos IS-LM como lo expone en la primera parte de su trabajo Romer (2000), muestra los principales problemas que adolece el modelo, por ejemplo: i) la ausencia de microfundamentos, ii) ausencia del rol de las expectativas, iii) simplicidad de la complejidad de la economía y iv) presupone que el Banco Central establece una oferta monetaria fija. A raíz de estos problemas, Romer propone un modelo alternativo que modifique el supuesto del Banco Central que persigue un objetivo de oferta agregada por una propuesta de incluir que la autoridad monetaria siga una tasa de interés real. Asimismo, en el aporte de Bofinger et. al (2006) expone las tres ecuaciones fundamentales del modelo Neo keynesiano -demanda, oferta agregada y una regla de tasa de interés que sigue la autoridad monetaria- para la enseñanza de la economía monetaria, donde el Banco Central sigue un objetivo de metas de inflación a través de la regla de Taylor de tasa de interés simple. El trabajo Bofinger es una explicación estática de los modelos neokeynesiano básico, en cambio, la versión dinámica vendría a ser los modelos de equilibrio general dinámico (DSGE, por su sigla en inglés) que mediante la Función Impulso Respuesta (FIR, en adelante) expresan el comportamiento de las tres ecuaciones del modelo básico ante choques de oferta y demanda agregada. Está versión dinámica será la que desarrollaremos en este documento de análisis de reglas de tasas de interés de política monetaria.

El análisis de la política monetaria basado en los modelos DSGE muestra un gran avance en la literatura macroeconómica como estudios publicados por Clarida, Gali y Getler $(1999,2000)$, Woodford (2003), Gali (2008) y entre otras diversidades de textos de libro especializados en modelos dinámicos como la de Wickens (2014), quienes exponen la complejidad matemática y la extensión del análisis de política monetaria en economía abierta, en coordinación con la política fiscal, movimiento de capital, fricciones financieras y entre otras áreas de la economía para su tratamiento ante choques de oferta y demanda agregada. Sin embargo, en muchos casos se asumen como regla de política monetaria la regla simple de tasa de interés propuesto por Taylor (1993) por su simplicidad y empírico, pero se deja de lado el análisis de la diversidad de reglas de tasa de interés como lo exponen Clarida, Gali y Getler. 
Bajo estas consideraciones, el propósito del documento es evaluar la variedad de la familia de reglas de tasa de interés óptima y como éstas se ajustan ante shock de demanda y oferta agregada. El análisis del documento enfatiza sobre los aportes de Clarida, Gali y Getler (1999) de la elección de instrumentos de política de regla de tasa de interés óptima considerando información perfecta e imperfecta y otras reglas de tasas de interés basado en la aplicación empírica. Una explicación previo al análisis de la evaluación de las reglas de política, es acerca de las tres ecuaciones fundamentales de la política monetaria moderna, basado en microfundamentos que se deriva de una función de utilidad de agente representativo simple con dinero, la presencia de un continuo de empresas que producen cualquier bien que maximizan sus beneficios y, que impera el supuesto de economía competitiva, a la que se añade algunas distorsiones esenciales: rigideces nominales, poder de monopolio, el problemas de información, y se consideran shocks de demanda y oferta en un modelo de equilibrio general dinámico y estocástico.

El documento se desarrolla de la siguiente manera: Capítulo1, se explica los elementos claves de un modelo Neo-keynesiano y sobre la elección de instrumentos. Capítulo 2, se explica acerca de las ecuaciones fundamentales neo-keynesiana. Capítulo 3, analiza el problema de la elección de instrumento de política monetaria con énfasis en el tratamiento de reglas de tasa de interés. Capítulo 4, primero se exponen un resumen de las ecuaciones log-linealizadas considerando que la política monetaria sigue una regla de tasa de interés, luego se realiza una simulación considerando la sugerencia de la literatura sobre los valores de los parámetros estructurales para su respectiva calibración para obtener las Funciones Impulso Respuesta. Capítulo 5, ponemos algunas limitaciones del modelo y consideraciones finales del documento de investigación. 


\section{Capítulo 1}

\section{Elementos claves del Enfoque Nuevo Keynesiano y la elección de instrumento}

\subsection{Elementos claves de la estructura Neo-Keynesiana}

Un aspecto importante previo al desarrollo de los fundamentos teóricos de la política monetaria en una perspectiva Neo Keynesiano (NK, en adelante), es necesario describir los elementos claves de la estructura de este enfoque, dado que será de utilidad en el análisis que desarrollaremos más adelante.

El enfoque NK de la política monetaria ha influenciado en los últimos años que proliferaron investigaciones en el área de macroeconomía. Se ha estructurado con el rigor teórico de la combinación de Real Bussines Ciclye (RBC por su sigla en inglés) y con ingredientes keynesiano como la competencia monopolística y rigideces nominales. Asimismo, se ha convertido como la base para la nueva generación de modelos que se desarrollan para los bancos centrales y cada vez más utilizados para la simulación y predicción de la economía.

Siguiendo a Gali (2007), las principales características del enfoque NK está vinculado con:

i) Adopta cualquier de las herramientas desarrolladas por la teoría RBC, incluyendo el sistema de modelos de equilibrio general dinámico y estocástico (DSGE, por su sigla en inglés), basados en la conducta optimizador de las familias y las empresas, expectativas racionales, vaciado de mercados, etc.

ii) Las empresas son modelados como competidores monopolistas, es decir, cada empresa enfrenta a un calendario de demanda de bien para el bien que producen y fija precios para ese bien para maximizar sus beneficios netos.

iii) Rigideces nominales que son el elemento clave del modelo y la principal fuente de la política monetaria no neutral. Generalmente introducen la forma de restricciones con la frecuencia de como las empresas y/o los trabajadores pueden ajustar sus precios y salarios, respectivamente ${ }^{1}$.

\footnotetext{
${ }^{1}$ Un ejemplo de la implicancia de las restricciones es que la decisión de fijar los precios y salarios se convierte en fordward-looking, dado que los agentes reconocen que los precios-salarios que fijan seguirán siendo efectivos más allá del periodo actual. Ver Gali (2007).
} 
iv) Hace hincapié en el componente endógeno de la política monetaria (reglas de política monetaria) y las implicaciones de este componente más que los efectos de los cambios exógenos en un instrumento de política monetaria.

v) Adicionalmente, el marco teórico NK puede utilizarse para evaluar la conveniencia de las reglas de política alternativa, así como para determinar la regla óptima, utilizando un criterio basado en el bienestar, basado en la maximización de una función de utilidad con agente de consumidor representativo de la economía (microfundamentos) y de esta manera es inmune a la crítica de Lucas.

Una vez expuesto los principales supuestos del enfoque NK, ahora podemos dar paso al siguiente apartado, donde desarrollaremos las ecuaciones fundamentales de la teoría de la política monetaria y el respectivo análisis, ya sea, discrecional o con reglas.

\subsection{Reglas versus discrecionalidad y la elección de instrumentos}

La elección de instrumentos reglas versus discreción se discuten en el trabajo pionero de Kydland y Prescott (1977), quienes señalan que una política es inconsistente debido a las expectativas racionales, lo cual no hay manera de que se pueda aplicar la teoría de control óptimo, dado que los agentes económicos intentarán anticipar a las acciones que emprenda el gobierno, por este motivo, no se puede lograr la maximización de una función de bienestar social. El modelo de estudio considera jugadores inteligentes, es decir, cuando el gobierno realiza el anuncio de su política que considera óptima, pero el agente privado se anticipa y hace cambios, lo cual no será óptimo lo que era antes. Por tanto, la diferencia entre la optimalidad exante y expost es conocida como la inconsistencia de la política económica. Entonces las políticas inconsistentes debilitan significativamente la credibilidad de las políticas anunciadas, además aseguran que las políticas discrecionales son incapaces de lograr un equilibrio óptimo, en cambio una política guiado por reglas estabiliza los precios.

De acuerdo con el aporte de Pool (1970), una adecuada elección de instrumentos de política amortigua los shocks ya sea de origen monetario o del sector real de la economía. En el caso de origen monetario es adecuado fijar una tasa de interés como instrumento de política y dejar que los agregados monetarios se determinen a las fluctuaciones del mercado, en cambio, si el shock es de origen de la demanda agregada lo correcto sería fijar el agregado monetario y dejar que la tasa de 
interés se determine exógenamente en el mercado ${ }^{2}$. Entonces la preocupación de la elección de instrumentos para Clarida, Gali y Getler surge a partir del problema de la política de elegir una trayectoria temporal para el instrumento de tasa de interés nominal $i_{t}$ que permita generar las rutas en el tiempo para las variables objetivo de la brecha del producto $x_{t}$ y de la inflación $\pi_{t}$, que maximicen la función objetivo-sujeta a la restricción de comportamiento. Entonces, para resolver dicho problema de política, siguen en la formulación tradicional de los clásicos Jan Tinbeargen (1952), Henri Theil (1961) en objetivos e instrumentos de política. El modelo muestra una diferencia importante respecto con los clásicos: Las variables objetivas dependen no solamente de la política actual sino también de las expectativas de la política futuras. La brecha de producto dependerá de la trayectoria futura de la tasa de interés y a su vez depende de la inflación actual y del comportamiento esperado de la brecha del producto futuro.

Un banco central puede seguir una política monetaria de compromiso, es decir, conducir su política monetaria bajo una regla de política; por otro lado, una política bajo discreción (sin seguir una regla de política). En este contexto, un banco central que opere bajo discreción elige la tasa de interés actual para reoptimizar en cada periodo. En cambio, bajo una regla se escoge un plan para la ruta de la tasa de interés que sigue para siempre. Además, bajo una política de discreción, el sector privado se forma sus expectativas tomando en cuenta como el banco central ajusta su política, dado que el banco central está libre de reoptimizar en cada periodo. A diferencia de una política bajo regla, es simplemente la vinculación a un compromiso que hace que el comportamiento de la política tienda hacia el equilibrio. Más adelante desarrollaremos acerca de la política óptima bajo discreción y reglas.

\footnotetext{
${ }^{2}$ Ver De Gregorio (2007)
} 


\section{Capítulo 2}

\section{Fundamento Teórico de la Política Monetaria}

Hay una variedad en la literatura económica que tratan sobre reglas de política monetaria moderna como Svensson (1997), Clarida, Gali y Getler (1999, 2000), McCallum(2002), Woodford (2003), Gali (2008), y entre otros, pero como ya hemos mencionado anteriormente, haremos hincapié en los aporte de Getler, Gali y Getler quienes proponen un modelo de teoría monetaria moderna que se basa en un modelo macroeconómico de equilibrio general dinámico con dinero, con competencia imperfecta y rigideces nominales. ${ }^{3}$ El modelo considera la política monetaria no neutral, es decir, afecta al sector real de la economía en el corto plazo mucho más que en la estructura del análisis del modelo IS-LM. Un aspecto relevante es que la conducta actual de la economía real en el corto plazo depende significativamente de las expectativas del curso futuro de la política monetaria más que de la política actual. Por simplicidad, el modelo se abstrae de la inversión y de la acumulación del capital. Esta abstracción no afecta a ninguna conclusión cualitativa.

Ahora, presentaremos un modelo de equilibrio general estocástico (DSGE, por su sigla en inglés) para una economía cerrada, donde se considera la participación de los agentes económicos de las empresas privadas, hogares, gobierno y un banco central. Este último es la autoridad encargada en implementar políticas bajo discreción o reglas, discusión que examinaremos más adelante cuando consideremos la tasa de interés nominal como instrumento de política monetaria. En este análisis se prescinde de la participación del gobierno por cuestiones de simplicidad de análisis, y que no causa distorsión alguna en los resultados.

\subsection{Hogares}

Primero, vamos a suponer que existe un gran número de agentes económicos de vida infinita en una economía cerrada. Entonces, se considera un modelo monetario simple con un hogar representativo con idénticas preferencias representado por la siguiente función de utilidad.

$$
\max E_{0} \sum_{t=0}^{\infty} \beta^{t} U\left(C_{t}, N_{t}, \frac{M_{t}}{P_{t}}\right)
$$

Sujeto a una restricción presupuestaria:

\footnotetext{
${ }^{3}$ El modelo NK no es derivado de un modelo de equilibrio general micro-fundamentado, aunque es un modelo de toda la economía.
} 


$$
P_{t} C_{t}+B_{t}+M_{t}=W_{t} N_{t}+\left(1+i_{t-1}\right) B_{t-1}+M_{t-1}+\Omega_{t}+T_{t}
$$

En el lado derecho de la ecuación (2.2) expresa la fuente de recursos con que cuenta la familia representativa, que provienen de ingresos laborales $W_{t} N_{t}$, ingresos por el rendimientos de sus activos (bonos) $\left(1+i_{t-1}\right) B_{t-1}$ del periodo $t-1$, saldos nominales con que cuenta la familia $M_{t-1}$ en el periodo $t-1$, ingresos por el beneficio empresarial $\Omega_{t}$ en el periodo $t$ y más las transferencias que reciben las familias $T_{t}$ en el periodo $t$. Por el lado de usos de los ingresos, las familias destinan en consumo real de bienes $P_{t} C_{t}$ en el periodo $t, B_{t}$ es el valor nominal de los bonos de portafolio en el periodo $t$, y los saldos nominales $M_{t}$ en el periodo $t$. Entonces, sustituyendo la ecuación (2.2) en (2.1), tenemos el lagrangiano:

$$
\mathcal{L}=E_{0}\left\{\sum_{t=0}^{\infty} \beta^{t} U\left(C_{t}, N_{t}, \frac{M_{t}}{P_{t}}\right)+\sum_{t=0}^{\infty} \beta^{t} \lambda_{t}\left[W_{t} N_{t}+\left(1+i_{t-1}\right) B_{t-1}+M_{t-1}+\Omega_{t}+T_{t}-P_{t} C_{t}-B_{t}-M_{t}\right]\right\}
$$

Aplicado la Condición de Primero Orden (CPO, en adelante) se exponen los resultados en la siguiente tabla:

Tabla 1: Ecuaciones de Primer Orden

$\begin{aligned} C_{t}: & U_{c, t} & =\lambda_{t} P_{t} \\ N_{t}: & -U_{N, t} & =\lambda_{t} W_{t} \\ M_{t}: & \lambda_{\mathrm{t}} & =U_{m, t} \frac{1}{P_{t}}+\beta E_{t}\left\{\lambda_{t+1}\right\} \\ B_{t}: & \lambda_{\mathrm{t}} & =\beta\left(1+i_{t}\right) E_{t}\left\{\lambda_{t+1}\right\}\end{aligned}$

En la Tabla 2, se expone los resultados de la combinación de las ecuaciones de primer orden, para obtener la función de oferta de trabajo (2.8), la ecuación de Euler del consumo (2.9) y la demanda de dinero (2.10). Por último, la combinación entre la ecuación (2.9) y (2.10) se obtiene la ecuación (2.11).

Tabla 2: Combinación de ecuaciones

\begin{tabular}{ll}
\hline \hline Combinación & \multicolumn{1}{c}{ Resultado } \\
\hline \hline (2.4) y (2.5) & $\frac{-\mathrm{U}_{\mathrm{N}, \mathrm{t}}}{\mathrm{U}_{\mathrm{c}, \mathrm{t}}}=\frac{\mathrm{W}_{\mathrm{t}}}{\mathrm{P}_{\mathrm{t}}}$ \\
$(2.4) \mathrm{y} \mathrm{(2.7)}$ & $\mathrm{U}_{\mathrm{c}, \mathrm{t}}=\beta\left(1+\mathrm{i}_{\mathrm{t}}\right) \mathrm{E}_{\mathrm{t}}\left\{\mathrm{U}_{\mathrm{c}, \mathrm{t}+1} \frac{\mathrm{P}_{\mathrm{t}}}{\mathrm{P}_{\mathrm{t}+1}}\right\}$ \\
$(2.4) \mathrm{y}(2.6)$ & $U_{c, t}=U_{m, t}+\beta E_{t}\left\{U_{c, t+1} \frac{P_{t}}{P_{t+1}}\right\}$ \\
\hline \hline
\end{tabular}




$$
\text { (2.9) y (2.10) } \quad \frac{U_{m, t}}{U_{c, t}}=\frac{i_{t}}{\left(1+i_{t}\right)}
$$

Por tanto, consideremos una expresión de una función particular $U\left(C_{t}, N_{t}, \frac{M_{t}}{P_{t}}\right)=\frac{C_{t}^{1-\sigma}}{1-\sigma}-\frac{N_{t}^{1+\varphi}}{1-\varphi}+$ $\frac{\chi}{1-v}\left(\frac{M_{t}}{P_{t}}\right)^{1-v}$, donde $\sigma=-\frac{U^{\prime}(c)}{U^{\prime \prime}(c) c}$ es el coeficiente de la elasticidad intertemproal de sustitución del consumo entre diferentes fechas o también es la aversión relativa al riesgo. Por tanto, los valores de: $U_{c, t}=C_{t}^{-\sigma}, U_{N, t}=-N_{t}^{\varphi}$ y $U_{m, t}=\chi\left(\frac{M_{t}}{P_{t}}\right)^{-v}$. Entonces, reemplazando los valores específicos, obtenemos el valor de la oferta de trabajo de los hogares, ecuación de Euler del consumo y la demanda de dinero de los hogares:

\begin{aligned} \hline & Tabla 3: Ecuaciones de la economía \\ $C_{t}^{\sigma} N_{t}^{\varphi} & =\frac{W_{t}}{P_{t}} \\ \left(\frac{M_{t}}{P_{t}}\right)^{d} & =\chi^{\frac{1}{v}} C_{t}^{\frac{\sigma}{v}}\left[\frac{i_{t}}{\left(1+i_{t}\right)}\right]^{-\frac{1}{v}} \\ 1 & =E_{t}\left\{\beta\left(\frac{C_{t+1}}{C_{t}}\right)^{-\sigma} \frac{P_{t}}{P_{t+1}}\left(1+i_{t}\right)\right\}\end{aligned}$

Ahora supondremos como condición un equilibrio de competencia monopolística para los hogares descritos anteriormente. Se considera un índice de consumo tipo Dixit-Stiglitz (1977) que define como:

$$
C_{t}=\left[\int_{0}^{1} C_{t}(i)^{\frac{\varepsilon-1}{\varepsilon}} d i\right]^{\frac{\varepsilon}{\varepsilon-1}}
$$

Donde $C_{t}(i)$ indica el consumo de los hogares del bien $i$ en el periodo $t$ y $\varepsilon>1$ es la elasticidad de sustitución (constante) entre los diferentes bienes. Ahora, utilizando la ecuación de la CPO del consumo del bien $i$ se tiene lo siguiente:

$$
\frac{1}{C_{t}} C_{t}^{\frac{1}{\varepsilon}} C_{t}(i)^{\frac{1}{\varepsilon}}=\lambda_{t} P_{t}(i)
$$

Teniendo en cuenta que $\lambda_{t}=\frac{1}{P_{t} C_{t}}$, entonces se tiene lo siguiente: 


$$
C_{t}(i)=\left(\frac{P_{t}(i)}{P_{t}}\right)^{-\varepsilon} C_{t}
$$

Esta ecuación expresa la optimización de la distribución del gasto en consumo en diferentes bienes en el tiempo $t$ para minimizar el gasto total necesario de los hogares y con el fin de alcanzar un determinado valor de índice de consumo. Entonces, considerando el equilibrio de $Y_{t}(i)=C_{t}(i)$, $i \in[0,1]$, entonces finalmente se tiene la siguiente ecuación de demanda del bien $i$ de cada empresa.

$$
Y_{t}(i)=\left(\frac{P_{t}(i)}{P_{t}}\right)^{-\varepsilon} Y_{t}
$$

Aplicando la misma condición de equilibrio a la ecuación de Euler (2.14) obtendremos lo siguiente:

$$
1=E_{t}\left\{\beta\left(\frac{Y_{t+1}}{Y_{t}}\right)^{-\sigma} \frac{P_{t}}{P_{t+1}}\left(1+i_{t}\right)\right\}
$$

Siguiendo el método de Uhlig (1999) ${ }^{4}$ podemos expresar la log-linealización de la nueva ecuación de Euler $(3.19)^{5}$

$$
x_{t}=E_{t}\left\{x_{t+1}\right\}-\varpi\left\{i_{t}-E_{t}\left\{\pi_{t+1}\right\}\right\}+g_{t}
$$

Donde $x_{t}=y_{t}-y_{t}^{n}$ es la brecha del producto, $i_{t}$ es la tasa de interés nominal, $\pi_{t+1}$ es la expectativa de inflación para $t+1, \varpi=\frac{1}{\sigma}$ es la elasticidad de sustitución intertemporal, $E$ es el operador de esperanza y se añade un shock de demanda agregada $g_{t}$ que sigue un proceso autoregresivo de orden uno, con $N\left(0, \sigma_{\varepsilon}^{2}\right)^{6}$. Está ecuación expresa la nueva curva IS y es la demanda agregada de la economía. Más adelante será de utilidad cuando evaluaremos las reglas de tasa de interés óptimas de política monetaria.

\footnotetext{
${ }^{4}$ Para cualquier variable $X_{t}$ define la desviación en logaritmos en el periodo $t$ de un estado estacionario estacionario no estocástico como se detalla lo siguiente: $\hat{x}_{t}=\ln X_{t}-\ln \bar{X}$, entonces, $X_{t}=\bar{X} e^{\hat{x}_{t}}$.

${ }^{5}$ Ver Wickens (cap.13, 2014).

${ }^{6}$ Ver Gali (2008) y en el anexo se encuentra el desarrollo del proceso log-linealizado.
} 


\subsection{Las empresas y el precio óptimo}

Ahora expondremos la Nueva Curva de Phillips u Oferta Agregada de la economía, para ello, se asume un continuo de empresas indexados $i \in[0,1]$ de competencia monopolística y cada empresa produce bienes diferenciado para el cual establecen el precio. Se impone algunas limitaciones al mecanismo de ajuste de precio al asumir que sólo una fracción de las empresas pueden establecer su precio en un periodo determinado. En particular se adopta un modelo de ajuste de precios escalonados (staggered price) a la calvo (1983). Todas las empresas utilizan una tecnología idéntica, representada por la función de producción.

$$
Y_{t}(i)=A_{t} N_{t}(i)^{1-\alpha}
$$

Donde $Y_{t}(i)$ es el nivel de producción de la empresa que produce algún bien $i, A_{t}$ representa el nivel tecnológico y es una variable exógena en el tiempo, $N_{t}$ es la cantidad de trabajo que emplea la empresa para la producción de algún bien $i$ en el tiempo $t$, y $1-\alpha$ es el parámetro que mide el rendimiento del trabajo.

Una empresa re-optimiza en el periodo $t$ eligiendo el precio $P_{t}^{*}$ que maximiza el valor actual del beneficio generado en el mercado. Para ellos se tiene la siguiente ecuación de beneficio:

$$
\max _{P_{t}^{*}} \sum_{k=0}^{\infty} \theta^{k} E_{t}\left\{Q_{t, t+k}\left(P_{t}^{*} Y_{t+k / t}-\Psi_{t+k}\left(Y_{t+k / t}\right)\right)\right\}
$$

Sujeto a una restricción de demanda

$$
Y_{t+k / t}=\left(\frac{P_{t}^{*}}{P_{t+k}}\right)^{-\varepsilon} C_{t+k}
$$

Para $k=0,1,2, \ldots$, donde $Q_{t, t+k}=\beta^{k}\left(\frac{C_{t+k}}{C_{t}}\right)^{-\sigma}\left(\frac{P_{t}}{P_{t+k}}\right)$ es el factor de descuento estocástico para los pagos nominales, $\Psi_{t}(\cdot)$ es la función de costo y $Y_{t+k / t}$ es el nivel de producción en el periodo $t+k$ para una empresa que fija su precio en el periodo $t$.

Entonces, sustituyendo la ecuación de restricción $(3,23)$ en la ecuación objetivo $(3.22)$, se tiene la siguiente ecuación de beneficio a maximizar: 


$$
\max _{P_{t}^{*}} \sum_{k=0}^{\infty} \theta^{k} E_{t}\left\{Q_{t, t+k}\left(\frac{P_{t}^{* 1-\varepsilon}}{P_{t+k}^{-\varepsilon}} C_{t+k}-\Psi_{t+k}\left(\left(\frac{P_{t}^{*}}{P_{t+k}}\right)^{-\varepsilon} C_{t+k}\right)\right)\right\}
$$

Aplicando la condición de primero orden se obtiene el siguiente resultado7:

$$
P_{t}^{*}=\frac{\epsilon}{\epsilon-1} \frac{\sum_{k=0}^{\infty} \theta^{k} E_{t}\left\{Q_{t, t+k} Y_{t+k \mid t} \psi_{t+k \mid t}\right\}}{\sum_{k=0}^{\infty} \theta^{k} E_{t}\left\{Q_{t, t+k} Y_{t+k \mid t}\right\}}
$$

Donde $\psi_{t+k \mid t}=\Psi_{\mathrm{t}+\mathrm{k} / \mathrm{t}}^{\prime}$ es el costo marginal en el periodo $t+k$, cuando las empresas fijan su precio en el periodo $t$. En ausencia de rigidez de precios $(\theta=0)$ y elasticidad de demanda constante, el precio óptimo vendría a ser $P_{t}^{*}=\mathcal{M} \psi_{t / t}$, donde $\mathcal{M}=\frac{\varepsilon}{\varepsilon-1}$ que es el markup sobre los costos ${ }^{8}$.

La dinámica del nivel de precio en un ambiente de competencia monopolística las empresas tienen la capacidad de fijar el precio del bien que ofrecen con probabilidad $1-\theta$, en cualquier periodo dado, pero la presencia de ciertos costes de menú hace que las empresas se enfrenten a costos al cambiar de precios, por lo que esto se ajusta cada cierto periodo. Por tanto, la evolución del precio de la economía está determinada por:

$$
P_{t}=(1-\theta) P_{t}^{*}+\theta P_{t-1}
$$

Nuevamente, aplicando el mismo método de log-linealización a la ecuación (2.25) y realizando algunas operaciones algebraicas, podemos llegar a la nueva ecuación de la curva de Phillips o ecuación de oferta agregada.

$$
\pi_{t}=\lambda x_{t}+\beta E_{t}\left\{\pi_{t+1}\right\}+u_{t}
$$

Como sabemos $\pi_{t}$ es la tasa de inflación, $x_{t}$ es la brecha del producto, ambos en el periodo $t, \lambda$ es el peso relativo de la brecha del producto, $\beta$ es el factor de descuento que es la pendiente de la nueva curva de Phillips y es la que define el comportamiento de la oferta agregada, por ejemplo: si $\beta=1$ la curva de oferta agregada de largo plazo que es perfectamente inelástica (vertical) y cuando $\beta<1$ tenemos una curva de oferta agregada con pendiente positiva ${ }^{9}$, por último, se incluye una

\footnotetext{
${ }^{7}$ Ver Anexo A.

${ }^{8}$ Considerando que el markup es endógeno que depende no solo de los valores de ciertas variables sino también de los valores esperado de las variables futuras.

${ }^{9}$ Ver Rodríguez (2008) y Bofinger (2006).
} 
variable aleatoria $u_{t}$ que expresa el shock de oferta, ha igual que el shock de demanda sigue un proceso autoregresivo de orden uno y con una distribución $N\left(0, \sigma_{\varepsilon}^{2}\right)$. 


\section{Capítulo 3}

\section{Evaluación de Regla de Tasa de Interés Óptima}

Previo a la evaluación de reglas de tasa de interés es necesario aclarar que normalmente los hacedores de política tradicional se guían por una función de pérdida social que normalmente minimizan la brecha y la inflación, sin embargo, en una perspectiva de la política económica moderna, el Banco Central se seguía por una función de bienestar social a maximizar que recoge las desviaciones respecto de sus variables objetivos para la elección de política ${ }^{10}$. A continuación, se expresa como:

$$
\max -\frac{1}{2} E_{t}\left\{\sum_{i=0}^{\infty} \beta^{i}\left[\alpha_{x} x_{t+i}^{2}+\pi_{t+i}^{2}\right]\right\}
$$

Donde $x_{t}$ y $\pi_{t}$ son los variables objetivos de política, $\alpha_{x}$ es un parámetro que expresa el peso relativo de las desviaciones del producto, dado que $x_{t}=y_{t}-y_{t}^{n}$, por tanto, la función considera como objetivo al producto potencial $y_{t}^{n}$, asimismo, implícitamente se mantiene cero la meta de inflación, pero no hay ningún costo en términos generales, dado que, la inflación es expresado como desviación porcentual de su tendencia.

\subsection{Política monetaria bajo discreción}

Una cuestión importante dentro del marco de la política monetaria es analizar los efectos bajo ausencia de compromiso (discreción). La virtud de este escenario se ajusta mejor con la realidad, es decir, que en la práctica ningún Banco Central hace cualquier tipo de compromiso vinculante en el transcurso de sus futuras políticas monetarias, y a su vez, comprender el rendimiento de la discrecionalidad. Entonces, bajo discreción, el Banco Central elige en cada periodo tres variables: la brecha del producto $\left(x_{t}\right)$, la inflación $\left(\pi_{t}\right)$ y la tasa de interés nominal $\left(i_{t}\right)$, las dos primeras son variables objetivos y el ultimo es el instrumento de política para maximizar la función objetivo de la ecuación (3.1), sujeto a la ecuación de la curva de oferta agregada y la curva IS. Para optimizar se divide en dos partes: primero el Banco Central elige $x_{t}$ y $\pi_{t}$ para maximizar el objetivo (2.1), dado la ecuación de inflación (curva de oferta agregada) (2.27), luego, dado la condición de los valores

\footnotetext{
${ }^{10}$ Argandoña (1996, cap. 6)
} 
óptimos de $x_{t}$ y $\pi_{t}$ se determina el valor óptimo de $i_{t}$, implícito en la curva IS (2.20). ${ }^{11}$ De esta manera se expresa como:

$$
-\frac{1}{2}\left[\alpha_{x} x_{t}^{2}+\pi_{t}^{2}\right]+F
$$

Sujeto a la siguiente restricción:

$$
\pi_{t}=\lambda x_{t}+f_{t}
$$

Donde $F_{t}=-\frac{1}{2} E_{t}\left\{\sum_{i=1}^{\infty} \beta^{i}\left[\alpha_{x} x_{t+i}^{2}+\pi_{t+i}^{2}\right]\right\}$ y $f_{t}=\beta E_{t} \pi_{t+1}+u_{t}$. Bajo discreción supondremos dos cosas: i) que el producto y la inflación futura no afectan a las acciones de hoy y ii) el Banco Central no puede manipular directamente las expectativas. Entonces, podemos escribir el lagrangiano de la siguiente manera ${ }^{12}$.

$$
\max _{\left\{x_{t}, \pi_{t}\right\}}-\frac{1}{2}\left[\alpha_{x} x_{t}^{2}+\pi_{t}^{2}+\phi\left(\pi_{t}-\lambda x_{t}\right)\right]
$$

Aplicando derivadas a la ecuación (3.4) respecto a la brecha del producto $x_{t}$ y a la inflación $\pi_{t}$ se llega a la siguiente condición óptima:

$$
x=-\frac{\lambda}{\alpha_{x}} \pi_{t}
$$

Esta condición simplemente implica que cuando la inflación esté sobre su objetivo, la demanda se contrae por debajo de su capacidad (por incremento en la tasa de interés); y viceversa, cuando está por debajo de su objetivo. La agresividad con la que el banco central debe reducir $x_{t}$ depende positivamente sobre los beneficios en la reducción de la inflación por unidad de perdida de la producción $\lambda$, e inversamente del peso relativo colocado en pérdidas de producción $\alpha$. Ahora, siguiendo con los mecanismos algebraicos, combinamos la condición óptima con la ecuación oferta agregada para obtener la forma reducida de las expresiones $x_{t}$ y $\pi_{t}$.

\footnotetext{
${ }^{11}$ En este caso la tasa de interés nominal ingresa como soporte a $x_{t}$ y $\pi_{t}$. Dado que el banco central no puede manipular de manera creíble bajo el escenario de la ausencia de compromiso, por lo que la autoridad monetaria toma como dado las expectativas del sector privado en la solución del problema de optimización. Más de esto se puede ver en Clarida et. al. (1999).

${ }^{12}$ Ver anexo B.
} 


$\begin{aligned} x_{t} & =-\lambda q u_{t} \\ \pi_{t} & =\alpha_{x} q u_{t} \\ i_{t} & =\gamma_{\pi} E_{t} \pi_{t+1}+\frac{1}{\varpi} g_{t}\end{aligned}$

Donde $q=\frac{\lambda}{\lambda^{2}+\alpha_{x}(1-\beta \rho)}$. La tasa de interés óptima (3.8) que expresa la política de retroalimentación, es el resultado de la sustitución del valor óptimo de la brecha del producto $x_{t}$ en la curva IS, donde $\gamma_{\pi}=1+\frac{(1-\rho) \lambda}{\rho \varpi \alpha_{x}}>1, E_{t} \pi_{t+1}=\rho \pi_{t}=\rho \alpha_{x} q u_{t}$.

Esta es la forma completa descrita de la política óptima, y como un primer resultado se puede señalar que en la medida que la inflación de coste está presente, existe un trade-off a corto palzo entre inflación y la variabilidad de la producción. Por tanto, la política óptima se define como una respuesta a un aumento de la inflación esperada, pero la tasa nominal debe aumentar lo suficientemente para aumentar la tasa real, es decir, el estado óptimo para la tasa nominal es posible cuando el coeficiente de la inflación esperada debe ser al menos mayor que la unidad ${ }^{13}$. Viendo la ecuación (3.8) refleja la meta implícita de la política óptima. Cuando la inflación se encuentra por encima de la meta, la política óptima requiere un aumento de la tasa real para contraer la demanda. En este sentido, la política óptima requiere de un ajuste perfecto de la tasa de interés para amortiguar el shock de la demanda $\left(g_{t}\right)$, pero amortiguar perfectamente el shock hacia el producto potencial $\left(y_{t}^{n}\right)$ para poder mantener la tasa nominal constante.

En cambio, un shock del producto potencial tampoco obliga a un trade-off a corto plazo, pero requiere de una respuesta de política diferente que al anterior que acabamos de analizar. Por ejemplo, un aumento permanente de la productividad aumenta también la demanda, siendo un amortiguador perfectamente debido a un impacto sobre el ingreso permanente, como consecuencia no cambia la brecha del producto, a su vez, no hay cambios en la inflación, por lo que no hay razón de aumentar la tasa, a pesar del aumento en el producto. Bajo estas consideraciones, podemos señalar que la política monetaria tiene una tarea fundamental e importante de distinguir

\footnotetext{
${ }^{13}$ En el documento de Clarida, Gali y Getler, realizan un análisis comparativo del resultado sobre la transición del óptimo hacia la meta de inflación, para ellos, Marvin Goodfriend y Robert King (1997) argumentan en favor de meta de inflación extrema, en cambio, Svensson (1997a, b) y Laurence Ball (1997) argumentan que la convergencia gradual de la inflación es óptima, sin embargo, está ausente la inflación de coste en Goodfriend y King y mientras en Svensson y Ball es un factor en la estructura del análisis.
} 
las fuentes u origen de los shocks del ciclo económico. En un escenario como acabamos de analizar con perfecta información, esta tarea es fácil identificar la fuente del ciclo de la actividad económica ${ }^{14}$, y no así cuando se relaja estos supuestos que muestran ciertas complicaciones en el análisis. Más adelante expondremos reglas de política monetaria considerando imperfecta información en la economía con un ejercicio de simulación para ver el ajuste ante shock de oferta y demanda.

\subsection{Compromiso de la Política Monetaria}

\subsubsection{Política monetaria bajo compromiso con restricciones}

Para ilustrar las ganancias del compromiso, primero se restringe la forma de regla de política a la forma general usada en el equilibrio bajo discreción y se resuelve el óptimo con esta clase de reglas. En el equilibrio sin compromiso es óptimo para el banco central ajustar $x_{t}$ únicamente en respuesta al shock del coste exógeno $u_{t}$, consideremos una regla para la variable objetivo $x_{t}$ que es consistente con el shock fundamental $u_{t}$, de la siguiente manera:

$$
x_{t}^{c}=-\omega u_{t}
$$

Para todo $t$, donde $\omega>0$ es el coeficiente de la regla de retroalimentación (feedback) y $x_{t}^{c}$ denota el valor de $x_{t}$ condicionada sobre compromiso de la política. Notamos que la regla incluye el óptimo bajo discreción como un caso especial (es equivalente a $\omega=\lambda q$ ). Ahora combinando con la ecuación de la curva de Phillips, a su vez implica que la inflación bajo la regla $\pi_{t}^{c}$, es también una función lineal del shock de coste.

\begin{tabular}{l} 
Tabla 5: Nueva Curva de Phillips con restricciones \\
\hline$\pi_{t}^{c}=\lambda x_{t}^{c}+\beta E_{t} \pi_{t+i}^{c}+u_{t}$ \\
$\pi_{t}^{c}=E_{t} \sum_{i=0}^{\infty} \beta^{i}\left[\lambda x_{t+i}^{c}+u_{t+i}\right]$ \\
$\pi_{t}^{c}=E_{t} \sum_{i}^{\infty} \beta^{i}\left[-\lambda \omega u_{t+i}+u_{t+i}\right]$ \\
$\pi_{t}^{c}=\frac{1-\lambda \omega}{1-\beta \rho} u_{t}$
\end{tabular}

${ }^{14}$ Ver Clárida, Gali y Getler (1999). 
El problema para el Banco Central es la elección del valor óptimo del parámetro feedback $\omega$. Con relación al caso de la discreción, la capacidad de comprometerse con una política de retroalimentación proporciona al banco central con una mejora a corto plazo del trade-off inflaciónproducto, para tal fin, es posible expresar como la ecuación siguiente:

$$
\pi_{t}^{c}=\frac{\lambda}{1-\beta \rho} x_{t}^{c}+\frac{1}{1-\beta \rho} u_{t}
$$

En este caso una contracción porcentual en $x_{t}^{c}$ reduce $\pi_{t}^{c}$ en $\frac{\lambda}{1-\beta \rho}$. Bajo discreción, reduciendo $x_{t}$ en un porciento sólo produce una caída en $\pi_{t}$ de $\lambda<\frac{\lambda}{1-\beta \rho}$. Por tanto, una política comprometida se debe al impacto extra de la regla de política sobre curso futuro de la brecha de la producción. La elección de $\omega$ no solo afecta a $x_{t}$, también a las creencias de $x_{1+i}^{c}, i=1,2, \ldots$, ya que $E_{t} x_{t+i}^{c}=-\omega u_{t}$. Un banco central que se compromete a una dura regla de política (implica que $\omega$ sea alto), por ejemplo, es capaz de señalar con credibilidad que se mantendrá en el tiempo una agresiva respuesta a un shock de oferta persistente. Dado que la inflación depende sobre el curso futuro del exceso de demanda, el compromiso con la dura regla de política conduce a un aumento de la inflación por unidad de producto perdido, en relación con el caso de discreción. Para encontrar el valor óptimo de $\omega$ notamos primero $x_{t+i}^{c}$ y $\pi_{t+i}^{c}$ que son cada uno múltiplo constante de los shocks de coste $u_{t+i}$. En este caso, es posible expresar la función objetivo como un múltiplo del periodo $t$.

\section{Tabla 6: Función objetivo con restricción}

$$
\begin{aligned}
& \max -\frac{1}{2} E_{t}\left\{\sum_{i=0}^{\infty} \beta^{i}\left[\alpha\left(x_{t+i}^{c}\right)^{2}+\left(\pi_{t+i}^{c}\right)^{2}\right]\right\} \\
& \max -\frac{1}{2}\left[\alpha\left(x_{t}^{c}\right)^{2}+\left(\pi_{t}^{c}\right)^{2}\right] L_{t}
\end{aligned}
$$

Con $L_{t}=E_{t}\left\{\sum_{i}^{\infty} \beta^{i}\left(u_{t+i} / u_{t}\right)^{2}\right\}>0$. Entonces el problema es elegir $\omega$ para maximizar la ecuación (3.16) sujeto a la ecuación (3.14), como resultado se obtiene la condición óptima:

$$
x_{t}^{c}=-\frac{\lambda}{\alpha^{c}} \pi_{t}^{c}
$$


Donde $\alpha^{c}=\alpha(1-\beta \rho)<\alpha$. Para el Banco Central, el óptimo significa el compromiso a una regla para crear una mayor contracción de la producción en respuesta a las presiones inflacionarios y que surge de una relación entre inflación y producto. Específicamente el costo de producción para reducir la inflación disminuye de $\alpha$ a $\alpha^{c}$, ya que reduciendo la inflación a una cantidad dada (ceteris paribus) requiere una sola fracción de $(1-\beta \rho)$ de pérdida del producto en virtud de la discreción. El descenso en el costo efectivo de reducir la inflación, a su vez, induce a una respuesta política más agresiva a la inflación. Ahora, la solución del equilibrio para $x_{t}^{c}$ y $\pi_{t}^{c}$ es expresado como:

\section{Tabla 7: Valores óptimos con restricciones}

$$
\begin{aligned}
x_{t}^{c} & =-\lambda q^{c} u_{t} \\
\pi_{t}^{c} & =-\alpha^{c} q^{c} u_{t} \\
i & =\gamma_{\pi}^{c} E_{t} \pi_{t+i}+\frac{1}{\varpi} g_{t}
\end{aligned}
$$

Con $q^{c}=\frac{1}{\lambda^{2}+\alpha^{c}(1-\beta \rho)}$. La solución en este caso se asemeja perfectamente a la solución obtenida bajo discreción que surge cuando $\alpha$ es remplazado por $\alpha^{c}<\alpha$ en la función objetivo. De ahí se deduce que condicionada al valor del shock de coste $u_{t}$, la inflación es cercana al objetivo y al producto, lo cual es un resultado relativo bajo discreción. Por otro lado, un caso particular, cuando $k=0$, el banco central no prefiere tener producción por encima del nivel potencial, entonces la solución bajo compromiso se ajusta perfectamente a la solución que obtendrá un banco central con discreción, donde asigna a la inflación un mayor costo que el verdadero costo social. Una característica adicional e interesante de este caso con compromiso implica la conducta de la tasa de interés óptima. Este puede ser visto formalmente reemplazando simplemente $\alpha^{c}$ por $\alpha$ en la regla de tasa de interés bajo discreción para obtener la ecuación (3.20). Entonces, con $\gamma_{\pi}^{c}=1+\frac{(1-\rho) \lambda}{\rho \varpi \alpha^{c}}>$ $1+\frac{(1-\rho) \lambda}{\rho \varpi \alpha}=\gamma_{\pi}$, el banco central incrementa la tasa de interés nominal por un importe mayor en respuesta a un aumento de la inflación esperada.

\subsubsection{Política monetaria bajo compromiso sin restricciones}

En la primera parte se mantiene el problema para elegir un estado dependiente para $x_{t+i}$ y $\pi_{t+i}$ para maximizar la función objetivo (ecuación (3.1)) dado la ecuación de la curva de oferta agregada (2.27) y en cada periodo $t+i, i \geq 0$. Ahora ya no restringimos la elección de $x_{t}$ a depender del valor contemporáneo del shock (es decir, $u_{t}$ ), pero permitir que en lugar de reglas una función de toda la 
historia de los choques. Para encontrar la solución óptima global al problema de política cuadrática lineal bajo compromiso, se tiene lo siguiente:

$$
\max -\frac{1}{2} E_{t}\left\{\sum_{i=0}^{\infty} \beta^{i}\left[\alpha_{x} x_{t+i}^{2}+\pi_{t+i}^{2}+\phi_{t+i}\left(\pi_{t+i}-\lambda x_{t+i}-\beta \pi_{t+i+1}-u_{t+i}\right)\right]\right\}
$$

Donde $\frac{1}{2} \phi_{t+i}$ es el multiplicador (estado) asociado con la restricción $t+i$. Aplicando las condiciones del primer orden se tiene a continuación la condición óptima.

$$
x_{t+i}-x_{t+i-1}=-\frac{\lambda}{\alpha} \pi_{t+i}, \forall i=1,2,3, \ldots
$$

Entonces:

$$
x_{t}=-\frac{\lambda}{\alpha} \pi_{t}
$$

Recapitulando, bajo discreción en la política óptima, el Banco Central ajusta al nivel de la brecha del producto en repuesta a la inflación. La política óptima bajo compromiso requiere ahora ajustar al cambio en la brecha del producto en respuesta a la inflación. Un aspecto por destacar es cuando se implementa la política en el periodo inicial (es decir en $t$ ), el banco central debe simplemente ajustar al nivel de la brecha del producto $x_{t}$ en respuesta a la inflación $\pi_{t}$, como si fuera persiguiendo la política óptima bajo discreción, pero eso pasa sólo por un periodo ${ }^{15}$.

Para ver esto, Clarida, Gali y Getler hacen que la inflación $\pi_{t}$ dependa no solo de la brecha del producto $x_{t}$, sino, de las futuras expectativas de $x_{t+i}$. Entonces, suponer que hay un shock de costos que aumenta la inflación sobre la meta en el tiempo $t$. La respuesta óptima bajo discreción es reducir $x_{t}$, pero luego dejar $x_{t+i}$ que retorne a su tendencia, para que con el tiempo $\pi_{t+i}$ caiga de nuevo hacia la meta. Sin embargo, la política óptima bajo compromiso es seguir reduciendo $x_{t+i}$, mientras $\pi_{t+i}$ permanece sobre el objetivo. La amenaza (creíble) para continuar contrayendo $x_{t}$ en el futuro, a su vez, tiene el efecto inmediato a amortiguar la inflación actual (dado la dependencia de $\pi_{t}$ sobre los valores futuros de $x_{t}$ ). Con relación al caso de la discreción, el shock de costos tiene un pequeño impacto en la inflación actual.

Una desventaja de la política óptima sin restricciones bajo compromiso es que parece más complejo de implementar que la restringida. Como hemos visto, la regla restringida se asemeja en cada

\footnotetext{
${ }^{15}$ Por que $x_{t+i}$ en general depende de $x_{t+i-1}$, la política óptima (sin restricciones) bajo compromiso no es en general simplemente una función sobre las variables de estado contemporáneo $u_{t+i}$.
} 
dimensión de la política óptima bajo discreción, pero relativamente con más importancia en la lucha contra la inflación. En consecuencia, como ya hemos mencionado, es posible aproximar esta política bajo discreción con una selección adecuada del Banco Central. Sin embargo, no ocurre lo mismo, para la política óptima sin restricciones. Un Banco Central conservador que operan con discreción no tiene ningún incentivo obvio para fijarse a la regla de diferencias de la brecha del producto implícita en la ecuación (3.22).

Una ampliación ${ }^{16}$ es que la regla de tasa de interés que implementa la política óptima podría haber efectos secundarios indeseables. Para ver esto, se combina la ecuación (3.22) y (2.20) para obtener la regla implícita de la tasa de interés óptima.

$$
i=\vartheta_{\pi} E_{t} \pi_{t+1}+\frac{1}{\varpi} g_{t}
$$

Donde $\vartheta_{\pi}=\left(1-\frac{\lambda}{\alpha \varpi}\right)<1$. En consecuencia, bajo esta regla, un aumento anticipado de la inflación lleva a un descenso de la tasa de interés real. Si las presiones inflacionarias varían inversamente con la tasa real, entonces una regla de este tipo puede permitir fluctuaciones auto-satisfactorias en el producto y la inflación que son claramente sub-óptimas. Por tanto, la regla de política globalmente óptima bajo compromiso implica que el banco central ajusta parcialmente la demanda en respuesta a las presiones inflacionarias. Además, considerando un Banco Central conservador puede aumentar el bienestar bajo discreción. Con esta estrategia no parece posible alcanzar la regla globalmente óptima. Finalmente puede haber algunas complicaciones en la práctica en implementar la regla de tasa de interés globalmente óptima que involucra indeterminación potencial.

\subsection{Política monetaria empírica}

\subsubsection{Información imperfecta}

Hasta ahora se ha analizado la capacidad del Banco Central de controlar perfectamente las principales variables objetivos. En la práctica esto no siempre suele ocurrir, dado que existe información imperfecta de las variables macroeconómicas. En el momento en que se fija la tasa de interés, un banco central puede no tener la disponibilidad de toda la información relevante acerca del estado de la economía, es decir, ciertos datos toman tiempo a procesar y recolectar, asimismo,

${ }^{16}$ Discutido en Woodford (1998) 
las muestras son imperfectas, dado que el acceso a la información en tiempo real de algunas variables claves como el nivel natural de la producción son medidos con un margen de error.

Por tanto, en un escenario con información imperfecta se tiene varias implicaciones específicas:

- Primero, ya no es posible la especificación de simples reglas en términos de variables objetivos, como alternativa se usan metas intermedias que es directamente observable, ejemplo, el agregado monetario ${ }^{17}$.

- Segundo, se realizan elección de instrumentos de política no trivial, mientras, con información perfecta no importa si el banco central utiliza la tasa de interés de corto plazo o de un agregado monetario como instrumento de política, siempre y cuando que la función de demanda de dinero da una relación monótona entre estas dos variables. Con información imperfecta, la volatilidad ex-post de una variedad de variables claves de la elección de instrumento ${ }^{18}$.

\subsubsection{Pronóstico como objetivos intermedios}

Previamente al análisis de elección de instrumentos, consideremos la información imperfecta en el modelo sin compromiso, el Banco Central no puede observar los valores contemporáneos del producto, inflación o cualquier shock aleatorio. Entonces, sea $\Omega$ el conjunto de información de los bancos centrales en el momento en que fijan el tipo de interés que prevalece en el tiempo $t$. En este sentido, la condición óptima es expresada como.

$$
E\left\{x_{t} \mid \Omega_{t}\right\}=-\frac{\lambda}{\alpha} E\left\{\pi_{t} \mid \Omega_{t}\right\}
$$

Esta ecuación es similar a la versión del modelo de certidumbre con información perfecta expresado anteriormente, pero tomado en cuenta la información a priori que dispone la autoridad monetaria. Ahora, asumiendo la ausencia de correlación serial de los shocks de costos, es decir, $\rho=0$, así que

\footnotetext{
${ }^{17}$ La diferencia radica que cuando se tiene información perfecta una política puede ser expresado en términos equivalentes de metas o instrumentos ya que existe en términos generales una relación de uno a uno entre estas variables. En cambio, con información imperfecta, las reglas pueden ser expresados solo en términos de los respectivos pronósticos, como opuesto a los valores ex-post.

${ }^{18}$ Respecto al tema de elección de instrumentos para amortiguar efectos ya sean de shock de oferta o de demanda se debe seguir la propuesta de Willam Poole (1970), investigación que realiza un análisis de los instrumentos de política económica.
} 
$u_{t}=\hat{u}_{t}$, entonces, esto implica que los valores de equilibrio de las variables objetivos con información imperfecta, $x_{t}^{I}$ y $\pi_{t}^{I}$ son dados por:

Tabla 8: Valores óptimos con Información Imperfecta

$x_{t}^{I}=x_{t}+\left(\frac{\lambda}{\lambda^{2}+\alpha} \hat{u}_{t}+\hat{g}_{t}\right)=\hat{g}_{t}$
$\pi_{t}^{I}=\left(1+\frac{\lambda^{2}}{\alpha}\right) \pi_{t}+\lambda \hat{g}_{t}=\hat{u}_{t}+\lambda \hat{g}_{t}$

Donde $x_{t}$ y $\pi_{t}$ son los valores óptimos de las variables objetivos que surgen del caso de información perfecta, $\hat{u}_{t}$ y $\hat{g}_{t}$ son los shocks inesperados de costos y de demanda, respectivamente. La información imperfecta claramente involucra mayor volatilidad de la inflación, ya que el banco central no puede actuar inmediatamente para amortiguar el impacto del shock. El efecto sobre la volatilidad de la brecha de producción es algo difuso por la incapacidad de amortiguar el shock de demanda y esto hace que aumente la volatilidad del producto. Por otra parte, el banco central no puede amortiguar el impacto inflacionario del shock de costos, por lo que prioriza en reducir la volatilidad de la producción, sin embargo, hay una inequívoca reducción del bienestar ${ }^{19}$. Un aspecto adicional que destacar es que el shock de demanda ahora afecta al comportamiento del producto, un movimiento positivo a corto plazo entre inflación-producto se puede originar si $\hat{g}_{t}$ tiene una varianza suficientemente grande en comparación a $\hat{u}_{t} \cdot{ }^{20}$

\subsubsection{Problema de elección de instrumentos: Tasa de interés versus agregados monetarios}

A consecuencia de la información imperfecta es importante la elección de instrumentos de política, por lo que se expone dos alternativas: la tasa de interés versus agregados monetarios ${ }^{21}$. En la

${ }^{19}$ Donde $u_{t}$ esta serialmente no correlacionado: $x_{t}=\frac{-\lambda}{\lambda^{2}+\alpha} u_{t}$ y $\pi_{t}=\frac{\alpha}{\lambda^{2}+\alpha} u_{t}$. Ver: Clarida, Gali y Getler (1999).

${ }^{20}$ Generalizando el análisis para un escenario de información imperfecta derivada de los rezagos en la transmisión de la política monetaria, Clarida, Gali y Getler consideran $j$ periodos para un cambio de la tasa de interés actual para afectar el producto y otras $k$ periodos para un impacto en la inflación. En este sentido, en el lado izquierdo de la ecuación (4.35) aparecería $j$ periodos hacia adelante de pronóstico del producto y en el lado derecha sería $j+k$ periodos hacia adelante de pronóstico de inflación. Este caso es iportante, dado que la evidencia sugiere un rezago de 6 a 9 meses para el efecto de la tasa de interés en el producto y mientras el efecto de rezago en la inflación es de un año y medio.

${ }^{21}$ Los agregados monetarios son los elementos que integran la oferta monetaria u oferta de dinero. Los principales agregados monetarios son: $\mathrm{M} 1=$ disponibilidad de liquidez (moneda fraccionaria y billete de bancos), M2 = M1 más los saldos de los depósitos de ahorro, propiedad de los hogares y de las empresas no financieras de la economía. M3 = M1 + M2 y más saldos de los depósitos a plazo fijo, propiedad de los hogares 
práctica, el Banco Central ajusta la tasa overnight de los fondos prestable interbancarios para reunir los requerimientos de reserva. Esta tasa se controla para manipular la oferta de reserva bancarias, es decir, la cantidad de dinero de alto poder disponibles para satisfacer los requerimientos de la reserva bancaria. Ahora, el Banco Central debe establecer mecanismos o reglas mediante el uso de la siguiente ecuación de demanda de dinero, donde las reservas bancarias $m_{t}$ esta dado por:

$$
m_{t}-p_{t}=\varkappa y_{t}-\eta i_{t}+v_{t}
$$

Donde $p_{t}$ es el nivel de precio, $v_{t}$ es una variable aleatoria de la demanda de dinero. Ahora consideramos dos escenarios de análisis: i) si $v_{t}$ es perfectamente observable, entonces no importa si $i_{t}$ o $m_{t}$ es empleado como instrumento de política, dado la trayectoria en el tiempo de $i_{t}$ implícito por la política óptima, es posible retornar sobre la trayectoria del tiempo para $m_{t}$ que apoya esta política de la ecuación (3.28), ii) si $v_{t}$ no es observable, entonces la tasa de interés es el instrumento que permite al banco central ajustar el stock de dinero al shock de demanda, por tanto, no hay impacto del shock de demanda de dinero sobre el producto y la inflación, ya que, el banco central los acomoda perfectamente.

Ahora, asumimos por simplicidad la ausencia del shock de demanda y de costos $\left(g_{t}=0, u_{t}=0\right)$, de modo que el único shock es la innovación de la demanda de dinero. Entones, la tasa de interés implícita para un instrumento de oferta de dinero es dado por:

$$
i_{t}^{m}=i_{t}+\frac{1}{\eta+\varphi(\kappa+\lambda)} \hat{v}_{t}
$$

Donde $i_{t}$ es la tasa que surge bajo meta de tasa de interés y $\hat{v}_{t}$ expresan los movimientos inesperados en demanda de dinero. Un punto clave es que el shock de demanda de dinero puede inducir a una conducta volátil de la tasa de interés. Esto es verdad si la demanda de dinero es relativamente inelástica en el corto plazo, como ocurre en el caso de las reservas del banco. La volatilidad de la tasa de interés en el corto plazo alimentará la volatilidad del producto mediante la relación de la demanda agregada (ver ecuación 2.20). Por esta razón, que los bancos centrales usan la tasa de interés de préstamo interbancario como instrumento de política, una visión originalmente

y de las empresas no financiera de la economía. Y el M4 = M1 + M2 +M3 más los títulos públicos en poder del sector privado no financiero. Para la economía de México, Banxico define a M4 = M3 más la captación de las sucursales y agencias de bancos mexicanos en el exterior provenientes de residentes en el exterior y de nacionales. 
debido a Poole (1970). Lo analizado hasta aquí nos lleva al siguiente resultado: shocks no observables de la demanda de dinero producen una alta volatilidad de la tasa de interés cuando el agregado monetario es usado como el instrumento de política ${ }^{22}$, siendo la razón fundamental para preferir un instrumento de tasa de interés.

\subsubsection{Política conservadora: incertidumbre versus forward - looking}

En la práctica los Bancos Centrales ajustan sus modelos con mayor precaución de lo que el modelo predice $^{23}$. Un modelo incierto es un problema formidable. Idealmente se esperaría que el Banco Central estuviese continuamente aprendiendo sobre la economía y como ajusta su política a esta. Es posible ilustrar como un modelo con incertidumbre puede en principio introducir algunos grados de política precautoria. En concreto, suponer que hay dos parámetros del modelo que son variables aleatorias: la elasticidad de la tasa de interés en la ecuación IS y la pendiente de la brecha del producto en la curva de oferta agregada, ahora están dadas por $\widetilde{\varpi}=\varpi+\varepsilon_{t}$ y $\tilde{\lambda}=\lambda+\eta_{t}$. Asumir que $\varepsilon_{t}$ y $\eta_{t}$ son variables aleatorias que siguen una i.i.d. con media cero. En este caso, la condición para la política óptima se convierte:

$$
E_{t}\left\{x_{t} \mid \Omega_{t}\right\}=\frac{\lambda}{\alpha+\lambda^{2} \sigma_{\eta}^{2}} E\left\{\pi_{t} \mid \Omega_{t}\right\}+\left(\alpha+\lambda^{2}\right) \frac{\sigma_{\varepsilon}^{2}}{\varphi} r_{t}
$$

Donde $r_{t}=i_{t}-E\left\{x_{t+1} \mid \Omega_{t}\right\}$ es la tasa de interés real ex-ante. Bajo esta condición los parámetros con incertidumbre tal vez reduzcan la respuesta de los instrumentos de política a las perturbaciones

\footnotetext{
22 Pequeños agregados no son buen instrumento de política debido a la volatilidad de la tasa de interés implícita. Amplios agregados no son buenas metas intermedias debido a su relación inestable con la actividad agregada.

${ }^{23}$ Con precaución se refieren generalmente al "suavizamiento de tasas de interés". Para ser preciso una serie de autores muestran la regla de la política monetaria de la siguiente forma

$$
i_{t}=(1-\rho)\left[\alpha+\beta \pi_{t}+\gamma x_{t}\right]+\rho i_{t-1}+\varepsilon_{t}
$$

Donde $\alpha$ un parámetro que se interpreta como el estado estacionario de la tasa de interés nominal, $\rho \epsilon[0,1]$ es un parámetro que refleja el grado de pendencia de rezago de la tasa de interés. La tasa de interés está presente en distintos aspectos: i) la pendiente de los coeficientes estimados de la inflación y de la brecha del producto $\beta$ y $\gamma$ son típicamente pequeños que la regla óptima sugeriría. ii) hay un ajuste parcial de los movimientos de la $\pi_{t}$ y $x_{t}$, reflejado por la presencia de la tasa de interés rezagada en la regla ajustada. Es decir, $i_{t}$ es un promedio ponderado de algunos valores deseados que depende del estado de la economía ( $\left.\left[\alpha+\beta \pi_{t}+\gamma x_{t}\right]\right)$ y de la tasa de interés rezagada, donde el peso relativo depende del parámetro suavizado $\rho$ (los valores estimados para datos trimestrales son típicamente en el orden de 0.8 o 0.9 . lo que sugiere el ajuste lento en la práctica), que expresa de alguna manera el ajuste gradual de la tasa de interés.
} 
en la economía. Esto puede entonces motivar a una senda suavizada de la tasa de interés que es equivalente con certidumbre de la política aplicada.

\subsubsection{Reglas de tasa de interés bajo inflación endógena y producto persistente.}

Hasta ahora hemos considerado que la dinámica de la inflación y el producto se debió a un proceso de fuerzas exógenas. Ahora se considera una estructura alternativa que permita persistencia endógena en el producto e inflación. El propósito es mostrar que los resultados derivados de una estructura base se extiende a una estructura general. La mayor diferencia es que con la persistencia endógena en la inflación, el equilibrio de la política monetaria de retroalimentación ahora influye en la velocidad de convergencia de la inflación hacia su objetivo. Para ello, se considera la siguiente generalización de la curva IS y la oferta agregada.

Tabla 9: Demanda y Oferta con inflación endógena y producto persistente

$$
\begin{aligned}
& x_{t}=-\varpi\left[i_{t}-E_{t} \pi_{t+1}\right]+\theta x_{t-1}+(1-\theta) E_{t} x_{t+1}+g_{t} \\
& \pi_{t}=\lambda x_{t}+\phi \pi_{t-1}+(1-\phi) \beta E_{t} \pi_{t+1}+u_{t}
\end{aligned}
$$

La primera ecuación incorpora la brecha del producto rezagada un periodo en la curva IS, la segunda ecuación, agrega la inflación rezagada a la curva de oferta agregada. Los parámetros $\theta$ y $\phi$ miden la influencia del rezago versus las variables futuro esperado. Cuando $\theta=0$ y $\phi=0$ se tiene el modelo base, en cambio, $\operatorname{con} \theta=1$ y $\phi=1$ el modelo vuelve a ser un backward looking. Para simplificar el análisis suponemos que las variables de perturbación $g_{t}$ y $u_{t}$ no se encuentran serialmente correlacionados. Entonces, la condición óptima que gobierna la política está dada por:

Tabla 10: Condición óptima de la brecha del producto

$x_{t}=-\frac{\lambda}{\alpha}\left[\pi_{t}+\sum_{k=1}^{\infty} \beta^{k} E_{t} \pi_{t+k}\right]$
$x_{t}=-\frac{\lambda}{\alpha\left(1-\beta a_{\pi}\right)} \pi_{t}$
$i_{t}=\gamma_{\pi} E_{t} \pi_{t+1}+\gamma_{x} x_{t-1}+\frac{1}{\varpi} g_{t}$


Con $\pi_{t}=a_{\pi} \pi_{t-1}+a_{u} u_{t}$ y $0<a_{\pi}<1^{24}$. La presencia de inercia hace que el ajuste de la actual política monetaria afecta a la trayectoria futura de la inflación. Como consecuencia, ahora la política responde no solo a la inflación actual, sino también a los pronósticos de la inflación en un futuro indefinido. La ecuación (3.35), expresa la regla de tasa de interés óptima, donde $\gamma_{\pi}=1+$ $\frac{\lambda\left(1-a_{\pi}\right)}{\varpi \alpha a_{\pi}\left(1-\beta a_{\pi}\right)}, \gamma_{x}=\frac{\theta}{\varpi} \mathrm{y} E_{t} \pi_{t+1}=a_{\pi} \pi_{t}$.

Los resultados obtenidos en una política bajo discreción con el modelo básico son ampliados también en el caso con producto endógeno e inflación persistente, además de permitir dependencia rezagada en producto e inflación, hay también justificaciones empíricas para incorporar retrasos en el efecto de la política. Entonces la condición de optimalidad se vuelve:

$$
E_{t}\left\{x_{t+1}\right\}=-\frac{\lambda}{\alpha\left(a-\beta a_{\pi}^{I}\right)} E_{t}\left\{\pi_{t+2}\right\}
$$

Donde $a_{\pi}^{I}$ mide la dependencia serial en la inflación para este caso y mantiene $0<a_{\pi}^{I}<1$. En lado izquierdo de la ecuación (3.36) refleja el retardo de un periodo del impacto de la política sobre el producto, mientras, en el lado derecho de la ecuación refleja dos periodos de retardos sobre la inflación. Entonces, debido al impacto retardado de la política, el Banco Central toma tanto la brecha del producto en $t, x_{t}$, y el pronóstico de la inflación en $t+1, E_{t}\left\{\pi_{t+1}\right\}$, como predeterminados a partir de $t$. Por tanto, la solución en términos de variables predeterminadas puede ser expresado como:

Tabla 11: Inflación y regla de tasa de interés como predeterminados

\begin{tabular}{l}
$E_{t}\left\{\pi_{t+2}\right\}=a_{\pi}^{I} E_{t}\left\{\pi_{t+1}\right\}$ \\
$i_{t}=\gamma_{\pi}^{I} E_{t} \pi_{t+1}+\gamma_{x} x_{t}^{*}$ \\
\hline$\left(^{*}\right)$ donde $\gamma_{\pi}^{I}=1+\frac{\left(\gamma_{\pi}^{-1) \beta}\right.}{a_{\pi}^{I}}>1$
\end{tabular}

${ }^{24}$ Donde $a_{\pi}$ y $a_{u}$ son función de los parámetros subyacentes $(\alpha, \lambda, \beta, \phi)$ y $a_{\pi}$ mide el grado de la inflación
persistente, es decir, mide la velocidad de convergencia de la inflación bajo la política óptima. La magnitud de
$a_{\pi}$ depende positivamente del grado de inflación inercial $\phi$. En el caso del modelo base la inflación inercial es
igual a cero $(\phi=0)$ e implica $a_{\pi}=0$, por otro lado, $a_{\pi}$ también depende negativamente sobre la inflación
de costos relativo, medido por $\frac{1}{\alpha}$. Como en el caso base, si el disgusto por la inflación es alto ( $\alpha$ es baja), la
política óptima agresivamente contrae la demanda cuando la inflación está sobre la meta: Con persistencia
endógena, esta contracción no solo reduce la inflación sino también aumenta la velocidad de convergencia
hacia la meta. 


\subsubsection{Análisis de reglas simples}

Taylor (1993) propone una regla simple de tasa de interés en niveles, es decir, la tasa de interés nominal depende linealmente de la brecha de la inflación y del producto, tal propuesta, se puede expresar como la siguiente forma:

$$
i_{t}^{*}=\alpha+\gamma_{\pi}(\pi-\bar{\pi})+\gamma_{x} x_{t}
$$

Donde $\alpha=\bar{r}+\bar{\pi}, \gamma_{\pi}>1, \gamma_{x}>0$, $i_{t}^{*}$ es el objetivo de tasa de interés, $\bar{\pi}$ es la meta de inflación, $\bar{r}$ es la tasa de interés real de equilibrio de largo plazo. Sin embargo, Clarida, Gali y Getler estiman una regla de Taylor simple pero que recoge el efecto de la expectativa de la inflación futura para la política monetaria de la Reserva Federal de los Estados Unidos. Esta nueva formulación específica vendría a ser una versión forward looking de la regla de Taylor simple.

$$
i_{t}^{*}=\alpha+\gamma_{\pi}\left(E_{t} \pi_{t+1}-\overline{\bar{\pi}}\right)+\gamma_{x} x_{t}
$$

Bajo este modelo de regla de tasa de interés, vemos que la política monetaria responde a la inflación esperada que vendría ser contrario a la inflación rezagada, además esta formulación anida la sencilla regla de Taylor como un caso especial. Otro aspecto necesario en la práctica tiene que ver con aspectos cuantitativos que escapan de la esfera del análisis económico, por tanto, la ecuación (3.40) no es capaz de capturar el problema de correlación serial de los datos, para ello es importante suavizar el ajuste de la tasa de interés mediante la inclusión del término de rezago de la tasa de interés nominal, lo cual nos permite expresar de la siguiente manera:

$$
i_{t}=\rho i_{t-1}+(1-\rho) i_{t}^{*}
$$

En la lectura de Clarida, Gali y Getler, proponen reglas simples para objetivos como opuesto a instrumentos. De esta propuesta, el objetivo de inflación ha recibido la mayor atención. El nivel de precio como objetivo es otro tipo de regla simple que discute la literatura, sin embargo, no ha recibido mucho apoyo. Otra variable candidata a ser variable objetivo es el PIB nominal.

Una crítica importante que mencionar hacia la regla de interés simples, es que, bajo ciertas circunstancias, éstas pueden inducir inestabilidad. Es decir, en muchos modelos no puede haber un equilibrio determinado bajo parametrización de la política que gobierne. Sin embargo, hay un número infinito de combinaciones del dinero nominal y el nivel de precios que satisface esta 
condición de equilibrio. Respecto a esto, la regla de tasa de interés produce indeterminación nominal. Cuando hay un lento ajuste del precio, el problema de la indeterminación nominal desaparece. 


\section{Capítulo 4}

\section{Simulación del modelo}

\subsection{Resumen de las ecuaciones log-linealizada}

En esta sección se expone la forma reducida de las ecuaciones que representan el equilibrio de modelo entorno al estado estacionario no estocástico. Aplicando el método de Uhlig (1999) que define que para cualquier variable $X_{t}$ respecto a la desviación en logaritmos en el periodo $t$ de un estado estacionario no estocástico se obtiene la variable log-linealizada, es decir, $x_{t}=\ln X_{t}-\ln \bar{X}$,

entonces $X_{t}=\bar{X} e^{x_{t}}$. Como resultado de la aplicación del método se exponen en la siguiente Tabla:

Tabla 12: Ecuaciones log-linealizadas

\begin{tabular}{ll}
\hline \hline IS & $x_{t}=E_{t}\left\{x_{t+1}\right\}-\varpi\left(i_{t}-E_{t}\left\{\pi_{t+1}\right\}\right)+g_{t}$ \\
$m_{t}^{r}$ & $=\frac{\sigma}{v} x_{t}-\eta i_{t}+\hat{v}_{t}$ \\
$\pi_{t}$ & $=\lambda x_{t}+\beta E_{t}\left\{\pi_{t+1}\right\}+u_{t}$ \\
NCPNK & $y_{t}^{n}=\psi_{y}^{n} \hat{A}_{t}$ \\
Producto Potencial & $y_{t}=\hat{A}_{t}+(1-\alpha) N_{t}$ \\
Producto & $w_{t}=\left(\sigma+\frac{\varphi}{1-\alpha}\right) x_{t}-\frac{\varphi}{1-\alpha} \hat{A}_{t}$ \\
Salario real
\end{tabular}

Reglas de tasa de interés de Política Monetaria

\begin{tabular}{lll}
\hline \hline $\begin{array}{l}\text { Infl. Endógena y prod. } \\
\text { Persistente }\end{array}$ & $i_{t}=\gamma_{\pi} E_{t} \pi_{t+1}+\gamma_{x} x_{t-1}+\frac{1}{\varpi} g_{t}+v_{t}$ \\
Tasa de interés simple & $i_{t}=\phi_{\pi} \pi_{t}+\phi_{y} x_{t}+v_{t}$ \\
$\begin{array}{l}\text { Tasa de interés fordward - } \\
\text { looking } \\
\text { Tasa de interés suavizada } \\
\text { fordward-looking }\end{array}$ & $i_{t}=\phi_{\pi} E_{t} \pi_{t+1}+\phi_{y} x_{t}+v_{t}$ \\
\hline \hline Shocks de la economía & $i_{t}=\rho i_{t-1}+(1-\rho)\left(\phi_{\pi} \pi_{t+1}+\phi_{x} x_{t}\right)+v_{t}$ \\
\hline \hline Shock de Demanda & $g_{t}=\rho_{g} g_{t-1}+\varepsilon_{t}^{g}$
\end{tabular}




\begin{tabular}{ll}
\hline \hline Shock de Oferta & $u_{t}=\rho_{u} u_{t-1}+\varepsilon_{t}^{u}$ \\
Shock Tecnológico & $\hat{A}_{t}=\rho_{a} \hat{A}_{t-1}+\varepsilon_{\mathrm{t}}^{A}$ \\
Shock de tasa de interés & $v_{t}=\rho_{v} v_{t-1}+\varepsilon_{t}^{v}$ \\
\hline Clasificación de las Variables & $x_{t}, \pi_{t}, m_{t}^{r}, w_{t}, y_{t}^{n}, N_{t}, i_{t}$ \\
\hline \hline Variables endógenas & $g_{t}, u_{t}, \hat{A}_{t}, v_{t}$, \\
Innovaciones endógenas & $\varepsilon_{t}^{g}, \varepsilon_{t}^{u}, \varepsilon_{t}^{v}, \varepsilon_{\mathrm{t}}^{A}$ \\
Shocks exógenos
\end{tabular}

Fuente: elaboración en base a las ecuaciones del modelo teórico de la política monetaria. Nota: Los parámetros estructurales como $\eta=\frac{\beta}{v(1-\beta)}, \lambda=\frac{(1-\theta)(1-\theta \beta)}{\theta} \frac{\sigma(1-\alpha)+\varphi+\alpha}{1-\alpha+\alpha \epsilon}$ y $\psi_{y}^{n}=$ $\frac{1+\varphi}{\sigma(1-\alpha)+\varphi+\alpha}$, su calculo se realiza previo a la calibración del modelo.

\subsection{Calibración del modelo}

Un paso previo a la calibración es en listar los valores de los parámetros del modelo, muchas de ellas se basan de acuerdo con la propuesta de la literatura estándar de los modelos de equilibrio general. Para nuestro caso, nos basamos en los aportes de Gali (2008, cap. 4 y 5), quien realiza análisis de política monetaria en un modelo básico de los Nuevos Keynesianos. En este sentido, para el análisis se exponen los valores de los parámetros de las ecuaciones de Demanda Agregada (4.1), Oferta Agregada (4.3) y todas las ecuaciones de las reglas de tasa de interés de política monetaria.

Tabla 13: Lista de los parámetros del modelo

\begin{tabular}{|c|c|c|c|}
\hline Descripción & Parámetro & valor & Fuente \\
\hline Factor de descuento & $\beta$ & 0.99 & Gali (2008) \\
\hline $\begin{array}{l}\text { Coeficiente de aversión relativa al riesgo } \\
\text { constante del consumo }\end{array}$ & $\sigma$ & 1 & Gali (2008) \\
\hline $\begin{array}{l}\text { Coeficiente de elasticidad de sustitución del } \\
\text { trabajo }\end{array}$ & $\varphi$ & 1 & Gali (2008) \\
\hline $\begin{array}{l}\text { Coeficiente de aversión relativa al riesgo } \\
\text { constante de la demanda de dinero }\end{array}$ & $v$ & 1 & Gali (2008) \\
\hline Coeficiente de shock de la demanda de dinero & $\chi$ & 1 & Gali (2008) \\
\hline $\begin{array}{l}\text { Influencia de la tasa de interés en la demanda de } \\
\text { dinero }\end{array}$ & $\eta$ & 4 & Gali (2008) \\
\hline Ponderador de la participación del trabajo & $\alpha$ & $1 / 3$ & Gali (2008) \\
\hline Probabilidad de mantener el precio anterior & $\theta$ & $2 / 3$ & Gali (2008) \\
\hline Elasticidad de sustitución entre bienes & $\epsilon$ & 6 & Gali (2008) \\
\hline $\begin{array}{l}\text { Coeficiente de autocorrelación de los shocks de } \\
\text { demanda }\end{array}$ & $\rho_{g}$ & 0.90 & Gali (2008) \\
\hline $\begin{array}{l}\text { Coeficiente de autocorrelación de los shocks de } \\
\text { inflación de costos (oferta) }\end{array}$ & $\rho_{u}$ & 0.90 & Gali (2008) \\
\hline
\end{tabular}




$\begin{array}{llll}\begin{array}{l}\text { Coeficiente de autocorrelación de los shocks de } \\ \text { tecnología }\end{array} & \rho_{a} & 0.90 & \text { Gali (2008) } \\ \text { Coeficiente de autocorrelación de los shocks de } & \rho_{v} & 0.40 & \text { Gali (2008) }\end{array}$

la tasa de interés nominal.

Fuente: Elaboración en base a la literatura de Gali (2008)

En la Tabla 14, se exponen los valores de los parámetros de las reglas de tasa de interés de política monetaria, para los cuales, se basaron en estudios presentados por Clarida, Gali y Getler (1999), Gali (2008), Andrew Levin y Rchhild Moessner (2005). Este último, realiza una aplicación para los países de la zona del euro.

Tabla 14: Lista de los parámetros de la regla de Política Monetaria

\begin{tabular}{|c|c|c|c|c|c|}
\hline Descripción & Parámetro & valor & \multicolumn{3}{|l|}{ Fuente } \\
\hline Coeficiente de elasticidad de sustitución & $\rho$ & 0.68 & \multicolumn{3}{|c|}{ "Ver Clarida, Gali y Getler (1999) } \\
\hline $\begin{array}{l}\text { Influencia de la inflación en la tasa de interés } \\
\text { nominal }\end{array}$ & $\phi_{\pi}$ & 1.5 & \multicolumn{3}{|l|}{ Ver Galí (2008) } \\
\hline $\begin{array}{l}\text { Influencia del producto en la tasa de interés } \\
\text { nominal }\end{array}$ & $\phi_{x}$ & $0.5 / 4$ & \multicolumn{3}{|l|}{ Galí (2008) } \\
\hline $\begin{array}{l}\text { Influencia de la inflación esperada en la tasa de } \\
\text { interés }\end{array}$ & $\gamma_{\pi}$ & 1.5 & $\begin{array}{l}\text { Andrew Levin } \\
\text { Moessner (2005) }\end{array}$ & $y$ & Rchhild \\
\hline $\begin{array}{l}\text { Influencia del producto esperado en la tasa de } \\
\text { interés }\end{array}$ & $\gamma_{x}$ & 0.44 & $\begin{array}{l}\text { Andrew Levin } \\
\text { Moessner (2005) }\end{array}$ & $y$ & Rchhild \\
\hline
\end{tabular}

Fuente: Elaboración en base a la literatura macroeconómica

La elección de los parámetros es una característica principal del análisis, ya que debe representar características económicas y contribuir a la condición de Blanchard-Kahn. Como se presentan en los Tablas 13 y 14, de acuerdo con Galí (2008), Andrew Levin y Rchhild Moessner (2005), usamos una calibración de los parámetros del modelo que comúnmente se selecciona en la literatura. Dado que en nuestro modelo los períodos se interpretan como trimestres, el coeficiente de la brecha del producto debe dividirse entre 4.

\subsection{Análisis de Función Impulso Respuesta (FIR's)}

Una vez identificado los priores de los parámetros del modelo estructural, ahora daremos paso al análisis de las FIR's expuestos gráficamente, que es resultado de la calibración de un sistema de ecuaciones formado por la demanda, oferta agregada y una regla de tasa de interés. Esta última ecuación sufrirá modificaciones considerando las diferentes opciones de reglas de tasa de interés óptima propuesto por la literatura y expuesto en el Capítulo 3. Entonces el análisis se divide de acuerdo con los respectivos shocks de demanda, oferta y tasa de interés, además, considerando cambios en los parámetros de la brecha del producto y metas de inflación. A continuación, expondremos cada una de ellas. 


\section{a) Shocks de demanda.}

Considerando shock demanda, podemos apreciar las FIR's expuesto en el Gráfico No. 1, donde se exponen diferentes resultados de la dinámica del producto, oferta agregada y de la regla de tasa de interés. En el panel (a) con una regla de tasa de interés óptima de inflación endógena y producto persistente muestra comportamiento poco usual, es decir, que la brecha del producto no sufre ninguna modificación, al igual que la inflación, es decir, el shock de demanda es nulo en el tiempo, sin embargo, la tasa de interés óptima sufre un incremento hasta el $1 \%$ que luego converge paulatinamente a cero. En cambio, en el panel (b) con tasa de interés simple, ante shock de demanda, el producto se incrementa positivamente a igual que la inflación y la regla de tasa de interés, sin embargo, en el panel (c) con regla de tasa de interés forward-looking, las reacciones de las tres variables son similares que el anterior panel, es decir, una regla simple de tasa de interés tendría similar respuesta que la tasa de interés forward - looking ante un shocks de demanda. Por último, los resultados del panel (d) considera una regla de tasa de interés forward-looking suavizada, en que la brecha de la producción y la inflación sufren incrementos positivos a igual que la tasa de interés, pero ésta última variable muestra una reacción suavizada ante shock de demanda agregada, a diferencia del resto de reglas de tasa de interés.

\section{Gráfico No 1: Shock de demanda}

Panel (a) Regla de tasa de interés óptima: $\hat{l}_{t}=\gamma_{\pi} E_{t} \pi_{t+1}+\gamma_{x} x_{t-1}+\frac{1}{\varpi} \hat{g}_{t}+v_{t}$
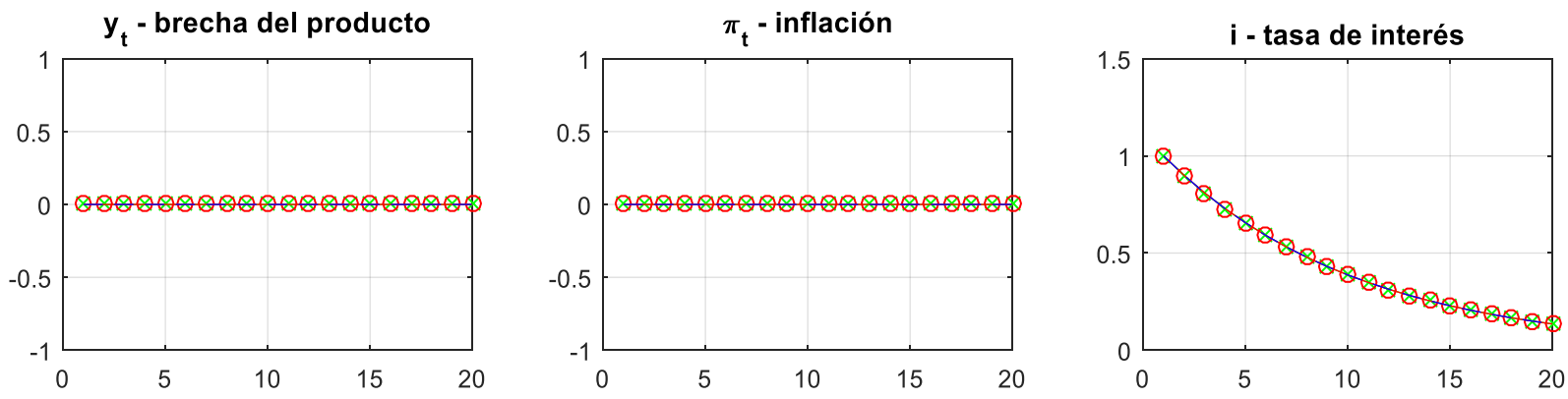

Panel (b) Regla de tasa de interés simple: $\hat{\imath}_{t}=\phi_{\pi} \pi_{t}+\phi_{y} \hat{y}_{t}+v_{t}$ 

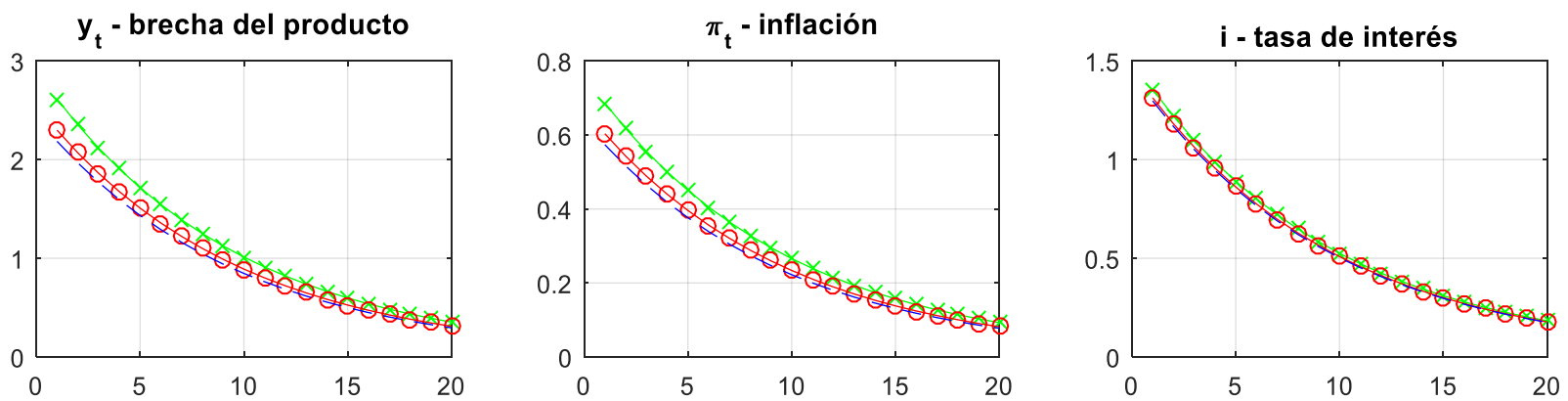

Panel (c) Regla de tasa de interés forward-looking: $\hat{\imath}_{t}=\phi_{\pi} E_{t} \pi_{t+1}+\phi_{y} \hat{y}_{t}+v_{t}$
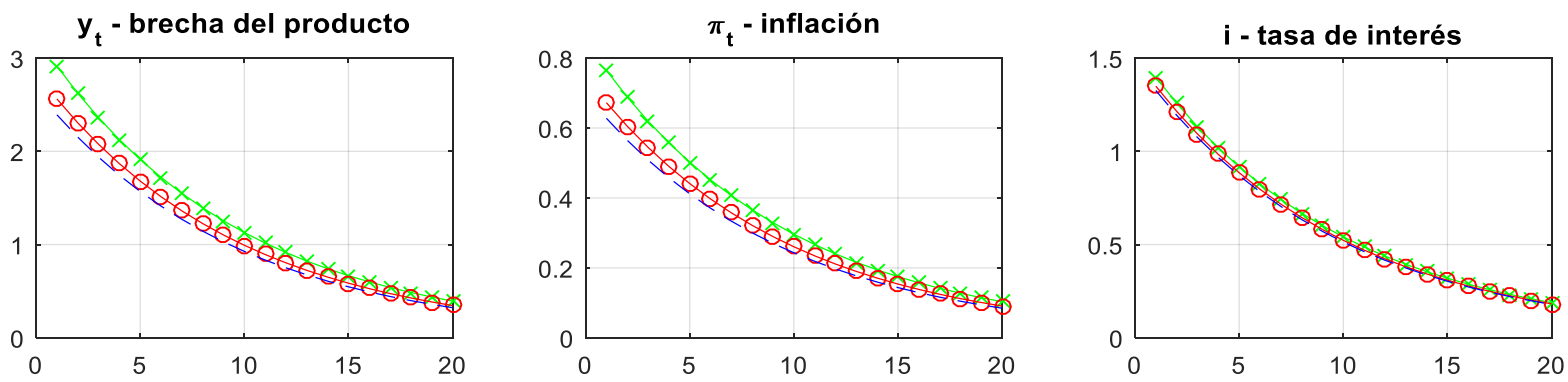

Panel (d), tasa de interés suavizada forward-looking: $\hat{\imath}_{t}=\rho i_{t-1}+(1-\rho)\left(\phi_{\pi} E_{t} \pi_{t+1}+\phi_{y} \hat{y}_{t}\right)+v_{t}$
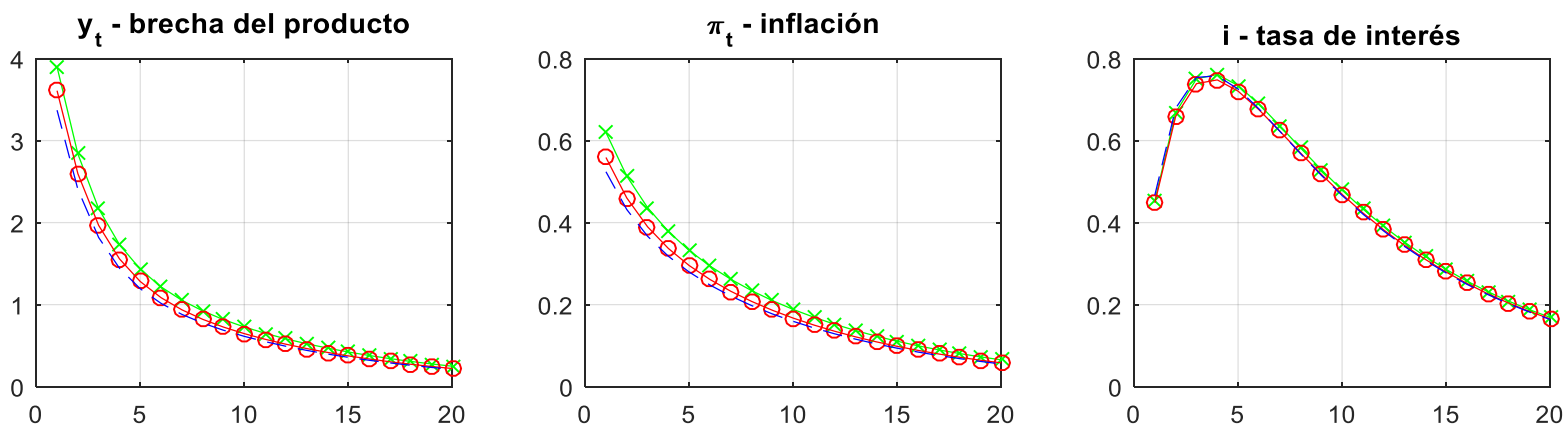

$\times$ Referencia $-O$ Mesta de inflación - - Brecha de producto

Fuente: Elaboración en base a los valores de los parámetros establecidos por la literatura macroeconómica

b) Shock de Oferta.

En un escenario de shock de oferta, se observa los resultados en la Gráfica No 2 donde muestran comportamientos concordantes con la teoría macroeconómica, por ejemplo, en los paneles: (a) con una tasa de interés de inflación endógena y producto persistente, (b) con tasa de interés simple y (c) tasa de interés forward-looking, muestran similares reacciones del producto, la inflación y de la tasa de interés ante un shock de oferta, es decir, en la brecha del producto, inflación y tasa de interés, provoca un desalineamiento transitorio respecto a su nivel potencial, meta de inflación y tasa de interés de largo plazo, respectivamente, ya que luego retornan paulatinamente a sus niveles 
de estado estacionario. En cambio, en el panel (d) con tasa de interés suavizada, los resultados son similares con las anteriores gráficas y por ende concordante con la teoría macroeconómica, sin embargo, hay un caso particular que es importante mencionarlas. Gráficamente, la respuesta del producto ante shock de oferta es más moderado que las anteriores gráficas, esto implicaría dentro del análisis de política monetaria y cuantitativa, que una regla suavizada resulta ser más amortiguador ante shock de inflación en costo a igual que la tasa de interés, ya que considera el efecto rezagado de un periodo de la tasa de interés nominal por lo que eliminaría la posible presencia de autocorrelación serial en los datos, asimismo, muestra que la política monetaria tiene efectos retardado en la actividad económica, por tanto al internalizar este efecto es más moderado las reacciones del producto y de la propia regla de tasa de interés.

\section{Gráfico No 2: Shock de Oferta}

Panel (a) Regla de tasa de interés óptima: $\hat{\imath}_{t}=\gamma_{\pi} E_{t} \pi_{t+1}+\gamma_{x} x_{t-1}+\frac{1}{\varpi} \hat{g}_{t}+v_{t}$
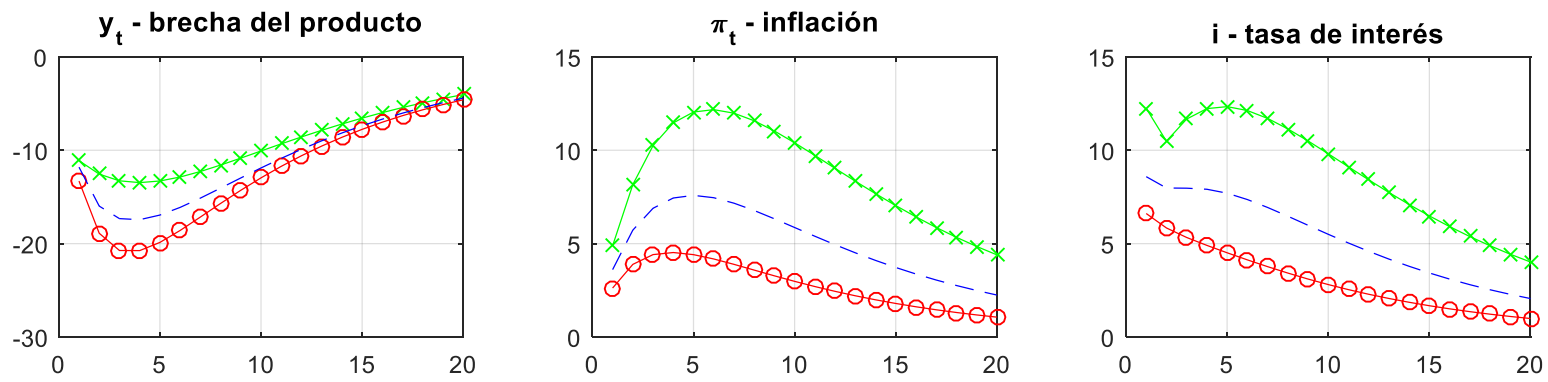

Panel (b) Regla de tasa de interés simple: $\hat{\imath}_{t}=\phi_{\pi} \pi_{t}+\phi_{y} \hat{y}_{t}+v_{t}$
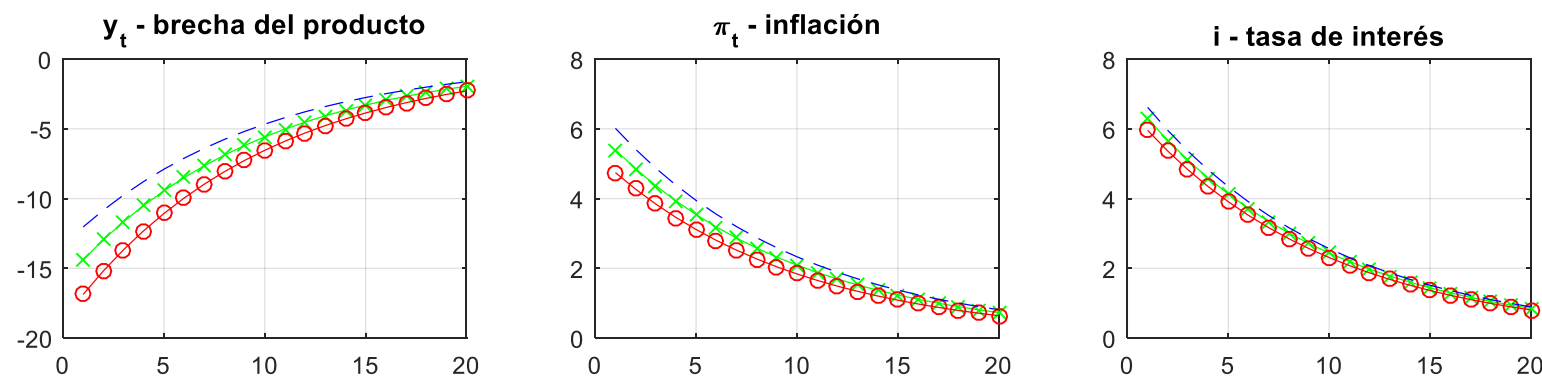

Panel (c) Regla de tasa de interés forward-looking: $\hat{\imath}_{t}=\phi_{\pi} E_{t} \pi_{t+1}+\phi_{y} \hat{y}_{t}+v_{t}$
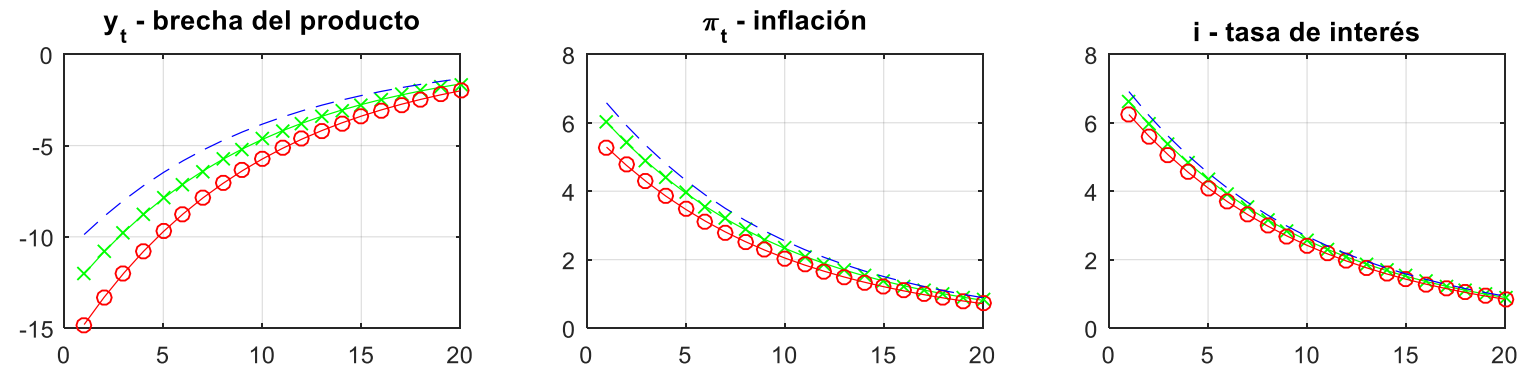
Panel (d), tasa de interés suavizada forward - looking: $\hat{\imath}_{t}=\rho i_{t-1}+(1-\rho)\left(\phi_{\pi} E_{t} \pi_{t+1}+\phi_{y} \hat{y}_{t}\right)+v_{t}$

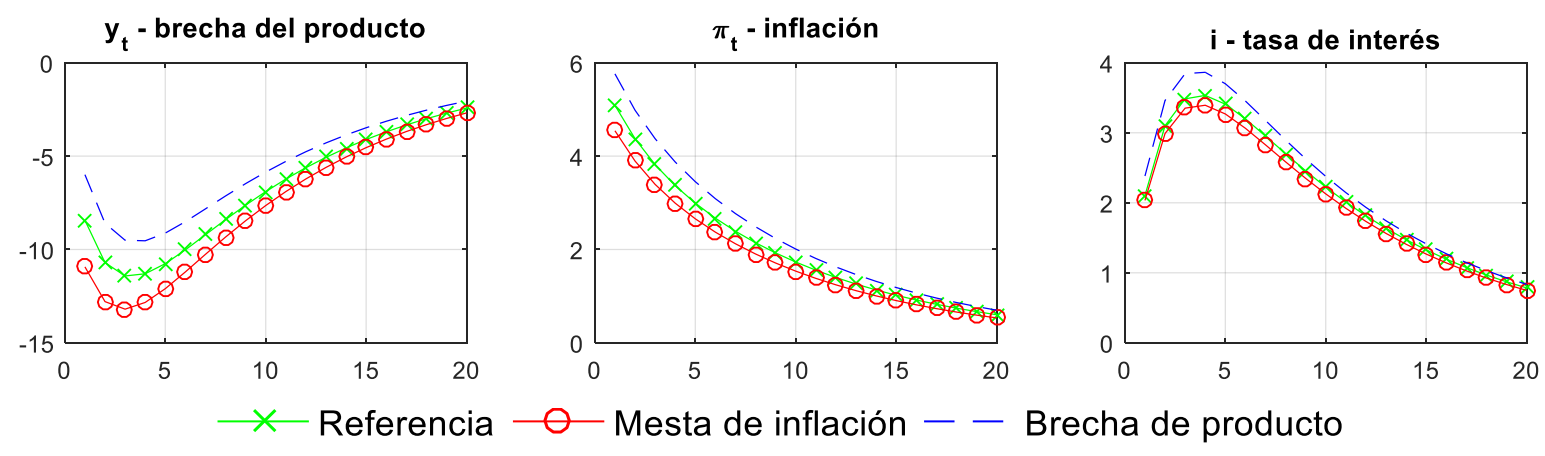

Fuente: Elaboración en base a los valores de los parámetros establecidos por la literatura macroeconómica

c) Shock de tasa de interés.

En el panel (a) de la gráfica No 3, podemos apreciar los resultados con tasa de interés de inflación endógena y producto persistente, los signos de reacciones de producto, inflación y tasa de interés ante un shock de tasa de interés pero que se diluye el efecto en cinco trimestres promedio, por lo que el efecto transitorio de una política monetaria contractiva impacta más a la inflación que al producto. Para el panel (b), se aprecia los resultados de las FIR's de la brecha del producto, inflación y tasa de interés, considerando una regla de tasa de interés de política monetaria simple, por lo que la respuesta del producto sufre un descenso en el nivel de producción, es decir, existe un alejamiento respecto a su nivel de producción potencial, sin embargo, este alejamiento no es duradero, ya que empieza converger desde el primero o segundo trimestre hacia su nivel potencial. De la misma manera, un shock de tasa de interés provoca una deflación transitoria en la economía porque luego converge a su nivel de meta de inflación, en cambio la tasa de interés sufre un aumento no duradero ya que luego de unos cinco trimestres se disipa el efecto del shock. En el Panel (c) considerando una regla de tasa de interés forward - looking se puede ver que las respuestas de las tres variables -brecha de producto, inflación y tasa de interés- son similares a los resultados expuestos en el panel (b). En cambio, con una regla de tasa de interés suavizada las tres variables reaccionan de manera abrupta ante shock de tasa de interés, esto podría asociarse con un posible problema de volatilidad del tipo de regla de tasa de interés considerado para este análisis. 


\section{Gráfico No 3: Shock de tasa de interés}

Panel (a) Regla de tasa de interés óptima: $\hat{\imath}_{t}=\gamma_{\pi} E_{t} \pi_{t+1}+\gamma_{x} x_{t-1}+\frac{1}{\varpi} \hat{g}_{t}+v$
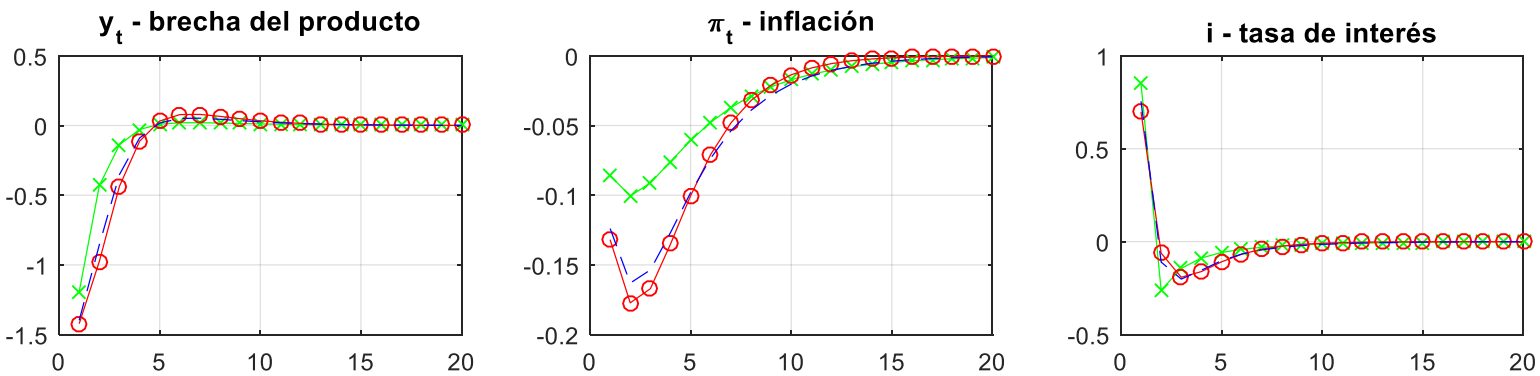

Panel (b) Regla de tasa de interés simple: $\hat{\imath}_{t}=\phi_{\pi} \pi_{t}+\phi_{y} \hat{y}_{t}+v_{t}$
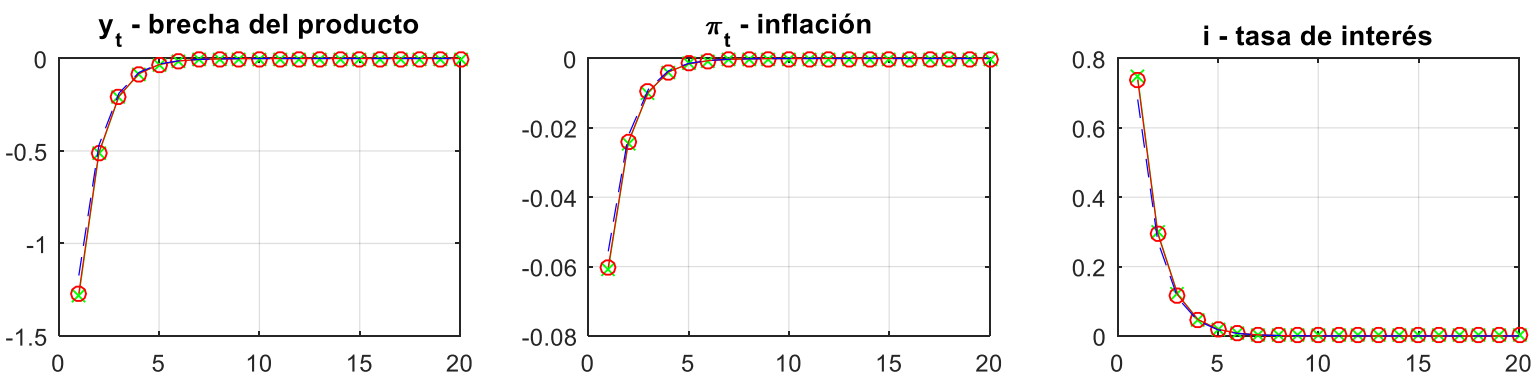

Panel (c) Regla de tasa de interés forward-looking: $\hat{\imath}_{t}=\phi_{\pi} E_{t} \pi_{t+1}+\phi_{y} \hat{y}_{t}+v_{t}$
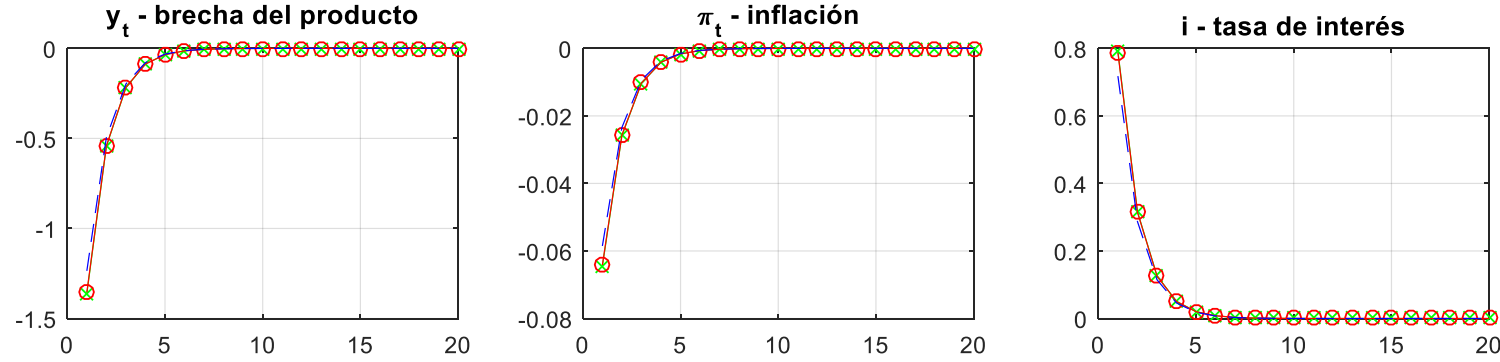

Panel (d), tasa de interés suavizada forward-looking: $\hat{\imath}_{t}=\rho i_{t-1}+(1-\rho)\left(\phi_{\pi} E_{t} \pi_{t+1}+\phi_{y} \hat{y}_{t}\right)+v_{t}$
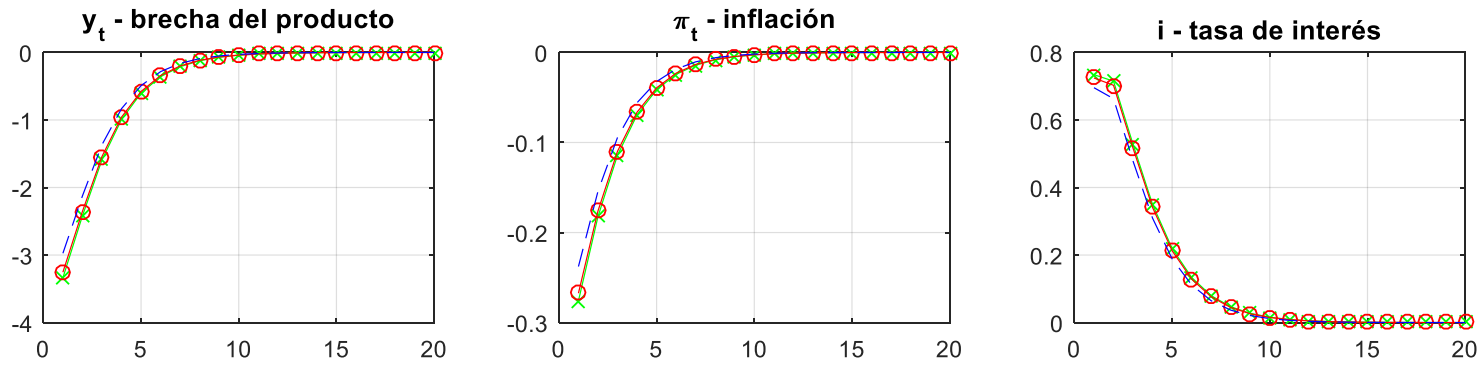

$\times$ Referencia - - Mesta de inflación - - Brecha de producto 


\section{Capítulo 5}

\section{Balances y Consideraciones finales}

\subsection{Limitaciones del modelo}

En el modelo neokeynesiano estándar, la política monetaria normalmente se caracteriza por una regla de Taylor tradicional, por lo cual la tasa de interés nominal se establece como un objetivo, sin embargo, en el aporte Clarida, Gali y Getler exponen la variedad de instrumento de política monetaria, como es el caso de la discrecionalidad, Compromiso (reglas) y algunas otras políticas empíricas (con reglas), que expresan cada una de ellas la manera de optimizar la política monetaria para amortiguar shocks inesperados de oferta y demanda agregada.

Con base al análisis que realiza Rodríguez (2008) sobre la elección de instrumentos de reglas versus discreción con presencia de manejo de tasa de interés y la nueva curva de Phillips, se puede distinguir los efectos de una política monetaria de estabilización de acuerdo con la forma de la pendiente de la curva de Phillips, es decir, $\beta=1$, hace que las autoridades monetarias manejen algunas tasas de interés como instrumento de política monetaria. Puesto que el producto y la inflación son variable no predeterminadas, cualquier shock de demanda se verá reflejado normalmente más en la inflación que en el producto, por tanto, será menos importante tener una política contracíclica para estabilizar la producción. En cambio, con presencia de expectativas bajo discrecionalidad puede dar origen a ciclos indeseados y el público espera que cambie los parámetros de la política monetaria. En esta situación las políticas con reglas estabilizan las expectativas y por ende el ciclo. Ante un shock de oferta, la demanda está imposibilitado en estabilizar el producto, por lo que lo único que provocaría una política contracíclica sería cambios en la inflación en dirección opuesta a como se está moviendo el producto. De aquí que para estabilizar las expectativas nuevamente, parece ser más deseable contar con reglas que actuar discrecionalmente.

Ahora supondremos en un escenario con $\beta<1$ (curva de Phillips con pendiente positiva). Ante shock de demanda sería posible estabilizar el producto mediante movimientos de la demanda, es decir, el uso de la política monetaria ya sea contractiva o expansiva. En caso de shock de oferta, el costo de estabilizar el producto con una política contracíclica es un efecto contrario de la inflación, es decir, si hay una reducción de la oferta el Banco Central puede implementar una política expansiva pero que generaría mayor inflación, a su vez, estabilizar la inflación propiciaría a exacerbar el efecto del shock de oferta. Entonces, en un caso de curva de Phillips con pendiente 
positiva, la política contracíclica de estabilización debe ser evaluada porque el uso de la discrecionalidad puede dar origen a ciclos generados por expectativas en el manejo de la demanda que son indeseables. En cambio, el uso de reglas minimiza los cambios súbitos de la política monetaria.

En todo caso, las políticas con reglas funcionan dentro de los límites de un modelo neokeynesiano simple, en que los efectos de la política monetaria se transmiten a través de la tasa de interés real. Gran parte de la literatura sobre política monetaria con reglas trata de supuestos con información perfecta e imperfecta y como amortiguar ante shock de oferta y demanda, sin embargo, en un contexto particular Zero Low Bond (ZLB, en adelante) que es un fenómeno de trampa de liquidez, la política monetaria expande a métodos no convencionales, que son efectivos en la medida en que el arbitraje del mercado financiero es imperfecto, que la autoridad monetaria asume el riesgo en su balance y/o la cantidad del dinero tiene un efecto en la economía independientemente de su efecto en la tasa de interés real. Estas políticas son interesantes y potencialmente útiles, pero el modelo neokeynesiano simple no es lo suficientemente complejo como para proporcionarles un $\mathrm{rol}^{25}$. Por otro lado, ante una situación de un fenómeno de trampa de liquidez, la literatura económica sugiere el uso de la política fiscal cuando la política monetaria pierde su eficacia, sin embargo, esto no es un tema de análisis en este documento.

Por último, podemos destacar las limitaciones del modelo DSGE aplicados en este documento para evidenciar el ajuste de las reglas óptimas de tasa de interés en la economía, en particular, en el comportamiento de la demanda y oferta agregada. De acuerdo con Blanchard (2016) hay muchas razones para sentirse en desacuerdo por los modelos DSGE:

- Primero, se basan en un supuesto poco arbitrario, no solo supuestos simplificadores, sino profundamente contrarios a los que sabemos de los consumidores y de las empresas, es decir, la demanda agregada se infiere de la demanda de consumo de consumidores previsivos que tienen una vida infinita. Sus implicaciones con respecto al grado de previsión y al papel de la tasa de interés en la modificación de la trayectoria del consumo están en fuerte discordancia con la evidencia empírica. El ajuste de precios es caracterizado por una ecuación de inflación futura que no capta la inercia fundamental de la inflación.

\footnotetext{
${ }^{25}$ Ver Betty Daniel (2017), quien sostiene que en un escenario ZLB la autoridad monetaria puede siempre encontrar algún instrumento estimulante. Este instrumento puede ser una política no convencional, aunque sostiene que puede ser un compromiso con una política de regla de Taylor.
} 
Segundo, el método de estimación y calibración no es convincente, dado que el policy maker en muchos casos realiza estimaciones por separadas o a veces los parámetros estructurales se fija como priores de acuerdo a la propuesta de la literatura económica, sin embargo, esto no es posible debido a las grandes diferencias en el comportamiento de las variables macroeconómicas entre países, por ejemplo, se suele utilizar "parámetro estándar de Calvo", los cuales determinan el efecto del desempleo en la inflación en países distintos, entonces, las decisiones de confiar en un conjunto estándar de parámetros es sólo una manera de trasladar a investigaciones anteriores la carga de elegir los parámetros.

\subsection{Conclusiones y perspectiva de la investigación}

De acuerdo con la literatura expuesta de Clarida, Gali y Getler, el documento intento evaluar las variedades de reglas de tasa de interés óptima, sin embargo, no muchas son posibles de aplicar en las decisiones de política monetaria, dado que son modelos teóricos de política monetaria derivados de una función de perdida social sujeto a restricciones de oferta y demanda agregada, bajo información perfecta. En cambio, en la realidad existe la presencia de información imperfecta, es decir, los Bancos Centrales no tienen la completa información de las principales variables macroeconómicas, ya que en muchos casos se demora el procesamiento de la información económica, en particular de aquellas variables relacionados con el sector real como el producto y entre otros, por tanto, la autoridad monetaria toma decisiones de acuerdo al conjunto de información disponible que cuenta en el periodo $t$, y en algunos casos realiza pronósticos de las variables objetivos siguiendo una reglas de tasa de interés simple propuesto por Taylor (1993), o también haciendo uso de reglas de tasa de interés suavizada que incluye el rezago de un periodo de la tasa de interés nominal que fija el banco central. Sin embargo, estas reglas empíricas como lo denominan Clarida, Gali y Getler, también presenta limitaciones al considerar un escenario de Zero Lower Bond (es decir, en presencia de la trampa de liquidez), donde la tasa se encuentra cercana o igual a cero (negativa en algunos casos), por lo que algunos estudios como Nakov (2006) analizan esta situación y proponen establecer una regla de Taylor simple truncado en cero que se incluye en el operador de maximización de la tasa de interés. En este mismo contexto, un reciente estudio de Betty Daniel (2017) propone una extensión de regla de tasa de interés considerando la implementación de una política monetaria óptima en el límite inferior cero, esto implica que la implementación de la política monetaria óptima en ZLB requiere centrarse en tres parámetros: el tiempo de salida, el objetivo de inflación al salir y la tasa de disminución de la meta de inflación. 
Por tanto, podemos resumir lo que aprendimos de esta este análisis de reglas de política de la siguiente manera. En primer lugar, con información perfecta las elecciones de instrumentos de política son triviales, además los ajustes de tasas de interés nominal óptima se pueden lograr mediante una elección cuidadosa de los argumentos dentro de la literatura que son muy sólidas. En segundo lugar, con información imperfecta ya no es posible especificar reglas simples en términos de variables objetivos por lo que se realizan la elección de instrumentos de política no triviales. Tercero, los modelos DSGE se basan excesivamente en el supuesto de mercados completos, considerando agentes económicos representativo, la existencia de un continuo de empresas que se deducen formalmente de microfundamentos. Normalmente estos modelos, se estiman como un sistema de ecuaciones y no ecuación por ecuación. Además, se pueden tratar de muchas maneras: teniendo en cuenta inversión, movimiento de capitales, la intermediación financiera, la interacción entre otros países, pero la ecuación de demanda y de oferta agregada siguen siendo fundamentales para el análisis de la economía.

Por último, a futuro como propósito de investigación sería necesario explorar marcos alternativos acerca de la evaluación de las reglas de tasas de interés de política monetaria para economías en desarrollo con política cambiaria, fricciones financieras y considerando otras reglas como los agregados monetarios McCallum (2002), reglas óptimas de política fiscal Woodford (2003), Gali (2008), ya que son de suma importancia que la literatura económica ha venido desarrollando en los últimos años. 


\section{Bibliografía}

Argandoña Antonio, G. C. (1996). Macroeconomía Avanzada I: Modelos Dinámicos y Teoría de Política Económica. Madrid: McGraw-Hill.

Blanchard, O. (2016). Do DSGE Model have a future? Peterson Institute for International Economics.

Bofinger, P. M. (2006). Th e BMW Model: A New Framework for Teaching Monetary Economics. Journal Economic Education vol. 37, no. 1, $98-117$.

Calvo, G. M. (1983). Staggered Prices in a Utility Maximization Framework. Journal of Monetary Economic, 383 - 398.

Clarida, R., Gali, J., \& Getler, M. (1999). The Science of Monetary Policy: A New Keynesian Perspective. Journal of Economic Literature, 1661 - 1707.

Daniel, B. (2017). Taylor - Rule exit policies for the zero lower bound. University at Albany - SUNY.

De Gregorio, J. (2007). Macroeconomía: Teoría y Política. Santiago - Chile: Pearson - Education.

Fontana, G., \& Stterfield, M. (2010). Macroeconomics theory and macroeconomic pedagogy. USA: Palgrave Macillan.

Gali, J. (2008). Monetary Policy, Inflation and the BusinessCycle: An Introduction to the New Keynesian Framework. New Jersey: Pricenton University Press.

Kydland, F., \& Prescott, E. (1977). Rules rather than discretion: the inconsistency of optimal plans. Journal of Political Economy, Vol. 85, $473-492$.

Levin, A., \& Moeesner, R. (2005). Inflation Persistence and Monetary Policy Design an Overview. Working Paper Series - European Central Bank, No. 539.

Nakova, A. (2008). Optimal and Simple Monetary Policy Rule with Zero Floor on the Nominal Interest Rate. International Journal of Central Banking, 4(2), 73 - 128.

Poole, W. (1970). Optimal Choice of Monetary Policy Intruments in a Sotchastic Macro Model. 84, $197-216$.

Pountineau, J. C., Sobczak, K., \& Vermandel, G. (2015). The analytics of the New Keynesian 3equation Model. Economics and Business Review, Vol. 1(15) No 2, $110-129$.

Rodriguez Arana Zumaya, A. (2008). Dinámica macroeconómica y precios rígidos: el papel de la política de estabilización. Ciudad de México: Universidad Iberoamericana.

Romer, D. (2000). Keynesian Macroeconomics the without LM curve. Journal of Economics Perspectives, Vol. 14, No. 2: 149 - 169.

Wickens, M. (2008). Macroeconomic Theory: A Dynamic General Equilibrium Approach. New Jersey: Princeton University Press.

Woodford, M. (2003). Interest and Prices: Foundations of a Theory of Monetary Policy. New Jersey: Princeton University Press. 


\section{Anexo}

A: Modelo de equilibrio general

Hogares

$$
\max E_{0} \sum_{t=0}^{\infty} \beta^{t} U\left(C_{t}, N_{t}, \frac{M_{t}}{P_{t}}\right)
$$

Sujeto a la restricción presupuestaria:

$$
P_{t} C_{t}+B_{t}+M_{t}=W_{t} N_{t}+\left(1+i_{t-1}\right) B_{t-1}+M_{t-1}+\Omega_{t}+T_{t}
$$

A continuación, se tiene la ecuación lagrangiano

$$
\mathcal{L}=E_{0}\left\{\sum_{t=0}^{\infty} \beta^{t} U\left(C_{t}, N_{t}, \frac{M_{t}}{P_{t}}\right)+\sum_{t=0}^{\infty} \beta^{t} \lambda_{t}\left[W_{t} N_{t}+\left(1+i_{t-1}\right) B_{t-1}+M_{t-1}+\Omega_{t}+T_{t}-P_{t} C_{t}-B_{t}-M_{t}\right]\right\}
$$

Aplicado la CPO:

Tabla A. 1: Ecuaciones de Primer Orden

\begin{tabular}{rlr}
\hline$C_{t}:$ & $U_{c, t}$ & $=\lambda_{t} P_{t}$
\end{tabular}

Ecuaciones combinadas:

Tabla A. 2: Resultado de las ecuaciones Combinadas

\begin{tabular}{lll}
\hline Combinación & \multicolumn{1}{c}{ Resultado } \\
\hline \hline (2.4) y (2.5) & $\frac{-\mathrm{U}_{\mathrm{N}, \mathrm{t}}}{\mathrm{U}_{\mathrm{c}, \mathrm{t}}}=\frac{\mathrm{W}_{\mathrm{t}}}{\mathrm{P}_{\mathrm{t}}}$ & (A. 8) \\
(2.4) y (2.7) & $\mathrm{U}_{\mathrm{c}, \mathrm{t}}=\beta\left(1+\mathrm{i}_{\mathrm{t}}\right) \mathrm{E}_{\mathrm{t}}\left\{\mathrm{U}_{\left.\mathrm{c}, \mathrm{t}+1 \frac{\mathrm{P}_{\mathrm{t}}}{\mathrm{P}_{\mathrm{t}+1}}\right\}}\right.$ \\
(2.4) y (2.6) & $U_{c, t}=U_{m, t}+\beta E_{t}\left\{U_{c, t+1} \frac{P_{t}}{P_{t+1}}\right\}$ \\
(2.9) y (2.10) & $\frac{U_{m, t}}{U_{c, t}}=\frac{i_{t}}{\left(1+i_{t}\right)}$
\end{tabular}


Función particular: $U\left(C_{t}, N_{t}, \frac{M_{t}}{P_{t}}\right)=\frac{C_{t}^{1-\sigma}}{1-\sigma}-\frac{N_{t}^{1+\varphi}}{1+\varphi}+\frac{\chi}{1-v}\left(\frac{M_{t}}{P_{t}}\right)^{1-v}$, entonces, los valores vendrían a ser: $U_{c, t}=C_{t}^{-\sigma}, U_{N, t}=-N_{t}^{\varphi}$ y $U_{m, t}=\chi\left(\frac{M_{t}}{P_{t}}\right)^{-v}$.

\begin{tabular}{|c|c|}
\hline$C_{t}^{\sigma} N_{t}^{\varphi}=\frac{W_{t}}{P_{t}}$ & (A. 12) \\
\hline$\left(\frac{M_{t}}{P_{t}}\right)^{d}=\chi^{\frac{1}{v}} C_{t}^{\frac{\sigma}{v}}\left[\frac{i_{t}}{\left(1+i_{t}\right)}\right]^{-\frac{1}{v}}$ & (A. 13) \\
\hline $1=E_{t}\left\{\beta\left(\frac{C_{t+1}}{C_{t}}\right)^{-\sigma} \frac{P_{t}}{P_{t+1}}\left(1+i_{t}\right)\right\}$ & (A. 14) \\
\hline
\end{tabular}

Índice consumo de los hogares

$$
\begin{gathered}
C_{t}=\left[\int_{0}^{1} C_{t}(i)^{\frac{\varepsilon-1}{\varepsilon}} d i\right]^{\frac{\varepsilon}{\varepsilon-1}} \\
C_{t}(i)=\left(\frac{P_{t}(i)}{P_{t}}\right)^{-\varepsilon} C_{t}
\end{gathered}
$$

Por la condición de equilibrio $Y_{t}(i)=C_{t}(i), \forall i \in[0,1]$ :

$$
Y_{t}(i)=\left(\frac{P_{t}(i)}{P_{t}}\right)^{-\varepsilon} Y_{t}
$$

\section{Derivada de la Curva IS:}

A partir de la ecuación (A.14) podemos obtener la ecuación de Euler log-linealizada.

$$
0=E_{t}\left\{-\sigma c_{t+1}+\sigma c_{t}+i_{t}-\pi_{t+1}\right\}
$$

Considerando que el producto del equilibrio $Y_{t}(i)=C_{t}(i)+E_{t}(i)$, donde $E_{t}(i)$ representa el gasto del gobierno en cualquier bien $i$, entonces log-linealizando $y_{t}=c_{t}+e_{t}$ :

$$
0=E_{t}\left\{-\sigma\left(y_{t+1}-e_{t+1}\right)+\sigma\left(y_{t}-e_{t}\right)+i_{t}-\pi_{t+1}\right\}
$$

Por definición la brecha del producto es $x_{t}=y_{t}-y_{t}^{n}$. Entonces reemplazando en la ecuación (A.19).

$$
0=E_{t}\left\{-\sigma\left(x_{t+1}+y_{t+1}^{n}-e_{t+1}\right)+\sigma\left(x_{t}+y_{t}^{n}-e_{t}\right)+i_{t}-\pi_{t+1}\right\}
$$




$$
y_{t}=E_{t}\left\{x_{t+1}\right\}-\frac{1}{\sigma}\left(i_{t}-E_{t}\left\{\pi_{t+1}\right\}\right)+E_{t}\left\{y_{t+1}^{n}-y_{t}^{n}\right\}-E_{t}\left\{e_{t+1}-e_{t}\right\}
$$

Si agrupamos a los dos últimos términos $g_{t}=E_{t}\left\{\Delta y_{t+1}^{n}\right\}-E_{t}\left\{\Delta e_{t+1}\right\}$, entonces obtenemos la curva IS o ecuación de demanda agregada tal cual expresada en el documento de Clarida, Gali y Getler (1999).

$$
x_{t}=E_{t}\left\{x_{t+1}\right\}-\frac{1}{\sigma}\left(i_{t}-E_{t}\left\{\pi_{t+1}\right\}\right)+g_{t}
$$

\section{Derivando la Curva LM:}

Hacemos uso de la ecuación (A.13) para log-linealizar:

$$
m_{t}^{r}=\frac{\sigma}{v} c_{t}-\frac{1}{v} \frac{\beta}{1-\beta} i_{t}
$$

Si asumimos que $\eta=\frac{1}{v} \frac{\beta}{1-\beta}$, podemos reescribir la ecuación anterior como:

$$
m_{t}^{r}=\frac{\sigma}{v} c_{t}-\eta i_{t}
$$

Ahora reemplazando a la ecuación (cc) la brecha del producto y considerando que el producto potencial $y_{t}^{n}=\psi_{y}^{n} \hat{A}_{t}$, entonces se obtiene la siguiente ecuación:

$$
m_{t}^{r}=\frac{\sigma}{v} x_{t}-\eta i_{t}+\frac{\sigma}{v} \psi_{y}^{n} \hat{A}_{t}-\frac{\sigma}{v} e_{t}
$$

Podemos considerar como un shock de demanda $\hat{v}_{t}=\frac{\sigma}{v} \psi_{y}^{n} \hat{A}_{t}-\frac{\sigma}{v} e_{t}$ :

$$
m_{t}^{r}=\frac{\sigma}{v} x_{t}-\eta i_{t}+\hat{v}_{t}
$$

\section{Derivando la ecuación de salario real:}

A partir de la ecuación (A.12) podemos aplicar el método de log-linealización, para la cual asumimos que $w_{t}=\ln W_{t}-\ln P_{t}$ para simplificar la operación algebraica.

$$
w_{t}=\sigma c_{t}+\varphi n_{t}
$$

Si el producto log-linealizado es: $y_{t}=\hat{A}_{t}+\left(1-\alpha_{N}\right) n_{t}$, y reemplazando en la ecuación anterior obtenemos la ecuación del salario real. 


$$
w_{t}=\left(\sigma+\frac{1}{\left(1-\alpha_{N}\right)}\right) y_{t}-\frac{\varphi}{\left(1-\alpha_{N}\right)} \hat{A}_{t}
$$

\section{Empresas}

Función de producción

$$
Y_{t}(i)=A_{t} N_{t}(i)^{1-\alpha}
$$

Se tiene una función de beneficio de la empresa

$$
\max _{\mathrm{P}_{\mathrm{t}}^{*}} \sum_{k=0}^{\infty} \theta^{k} E_{t}\left\{Q_{t, t+k}\left(P_{t}^{*} Y_{t+k / t}-\Psi_{t+k}\left(Y_{t+k / t}\right)\right)\right\}
$$

s. a.

$$
Y_{t+k / t}=\left(\frac{P_{t}^{*}}{P_{t+k}}\right)^{-\varepsilon} C_{t+k}
$$

donde el factor de descuento estocástico es $Q_{t, t+k}=\beta^{k}\left(\frac{C_{t+k}}{C_{t}}\right)^{-\sigma}\left(\frac{P_{t}}{P_{t+k}}\right)$, y reemplazando la ecuación de restricción en la función de beneficio.

$$
\max _{\mathrm{P}_{\mathrm{t}}^{*}} \sum_{k=0}^{\infty} \theta^{k} E_{t}\left\{Q_{t, t+k}\left(\frac{P_{t}^{* 1-\varepsilon}}{P_{t+k}^{-\varepsilon}} C_{t+k}-\Psi_{t+k}\left(\left(\frac{P_{t}^{*}}{P_{t+k}}\right)^{-\varepsilon} C_{t+k}\right)\right)\right\}
$$

Aplicando la condición de primer orden:

$$
P_{t}^{*}=\frac{\epsilon}{\epsilon-1} \frac{\sum_{k=0}^{\infty} \theta^{k} E_{t}\left\{Q_{t, t+k} Y_{t+k \mid t} \psi_{t+k \mid t}\right\}}{\sum_{k=0}^{\infty} \theta^{k} E_{t}\left\{Q_{t, t+k} Y_{t+k \mid t}\right\}}
$$

Ahora se considera un precio a la Calvo.

$$
P_{t}=(1-\theta) P_{t}^{*}+\theta P_{t-1}
$$

Realizando algunas operaciones a la ecuación (A.33):

$P_{t}^{*} \sum_{k=0}^{\infty} \theta^{k} E_{t}\left\{\beta^{k}\left(\frac{C_{t+k}}{C_{t}}\right)^{-\sigma} \frac{P_{t}}{P_{t+k}} Y_{t+k \mid t}\right\}=\frac{\epsilon}{\epsilon-1} \sum_{k=0}^{\infty} \theta^{k} E_{t}\left\{\beta^{k}\left(\frac{C_{t+k}}{C_{t}}\right)^{-\sigma} \frac{P_{t}}{P_{t+k}} Y_{t+k \mid t} \psi_{t+k \mid t}\right\}$ 
Aplicando el método de log-linealización

$$
\begin{aligned}
& \sum_{k=0}^{\infty} \theta^{k} E_{t}\left\{\bar{P} e^{\widehat{P} e_{t}^{t}} \beta^{k}\left(\frac{\bar{C} e^{\hat{C}_{t+k}}}{\bar{C} e^{\hat{C}_{t}}}\right)^{-\sigma} \frac{\bar{P} e^{\hat{P}_{t}}}{\bar{P} e^{\hat{P}_{t+k}}} \bar{Y} e^{\hat{P}_{t+k \mid t}}\right\}=\frac{\epsilon}{\epsilon-1} \sum_{k=0}^{\infty} \theta^{k} E_{t}\left\{\beta^{k}\left(\frac{\bar{C} e^{\hat{C}_{t+k}}}{\bar{C} e^{\hat{C}_{t}}}\right)^{-\sigma} \frac{\bar{P} e^{\hat{P}_{t}}}{\bar{P} e^{\hat{P}_{t+k}}} \bar{Y} e^{\hat{Y}_{t+k \mid t}} \bar{\psi} e^{\hat{\psi}_{t}+k \mid t}\right\} \\
& \overline{P Y} \sum_{k=0}^{\infty}(\theta \beta)^{k} E_{t}\left\{e^{\hat{P}_{t}^{t}-\sigma \hat{C}_{t+k}+\sigma \hat{C}_{t}+\hat{P}_{t}-\hat{P}_{t+k}+\hat{Y}_{t+k \mid t}}\right\}=\frac{\epsilon}{\epsilon-1} \overline{Y \psi} \sum_{k=0}^{\infty}(\theta \beta)^{k} E_{t}\left\{e^{-\sigma \hat{C}_{t+k}+\sigma \hat{C}_{t}+\hat{P}_{t}-\hat{P}_{t+k}+\hat{Y}_{t+k \mid t}+\hat{\psi}_{t}+k \mid t}\right\} \\
& \overline{P Y} \sum_{k=0}^{\infty}(\theta \beta)^{k} E_{t}\left\{1+\hat{P}_{t}^{*}-\sigma \hat{C}_{t+k}+\sigma \hat{C}_{t}+\hat{P}_{t}-\hat{P}_{t+k}+\hat{Y}_{t+k \mid t}\right\} \\
& =\frac{\epsilon}{\epsilon-1} \overline{Y \psi} \sum_{k=0}^{\infty}(\theta \beta)^{k} E_{t}\left\{1-\sigma \hat{C}_{t+k}+\sigma \hat{C}_{t}+\hat{P}_{t}-\hat{P}_{t+k}+\hat{Y}_{t+k \mid t}+\hat{\psi}_{t+k \mid t}\right\}
\end{aligned}
$$

Recordando el precio óptimo con perfecta flexibilidad de los precios $P_{t}^{*}=\mathcal{M} \psi_{t / t}$. Entonces la loglinealización vendría a ser: $\bar{P}=\frac{\epsilon}{\epsilon-1} \bar{\psi}$, ahora sustituyendo podemos simplificar la ecuación anterior:

$$
\begin{aligned}
& \sum_{k=0}^{\infty}(\theta \beta)^{k} E_{t}\left\{1+\hat{P}_{t}^{*}-\sigma \hat{C}_{t+k}+\sigma \hat{C}_{t}+\hat{P}_{t}-\hat{P}_{t+k}+\hat{Y}_{t+k \mid t}\right\} \\
& =\sum_{k=0}^{\infty}(\theta \beta)^{k} E_{t}\left\{1-\sigma \hat{C}_{t+k}+\sigma \hat{C}_{t}+\hat{P}_{t}-\hat{P}_{t+k}+\hat{Y}_{t+k \mid t}+\hat{\psi}_{t+k \mid t}\right\} \\
& 0=\sum_{k=0}^{\infty}(\theta \beta)^{k} E_{t}\left\{1-\sigma \hat{C}_{t+k}+\sigma \hat{C}_{t}+\hat{P}_{t}-\hat{P}_{t+k}+\hat{Y}_{t+k \mid t}+\hat{\psi}_{t+k \mid t}\right. \\
& \left.-1-\hat{P}_{t}^{*}+\sigma \hat{C}_{t+k}-\sigma \hat{C}_{t}-\hat{P}_{t}+\hat{P}_{t+k}-\hat{Y}_{t+k \mid t}\right\} \\
& 0=\sum_{k=0}^{\infty}(\theta \beta)^{k} E_{t}\left\{\hat{\psi}_{t+k \mid t}-\hat{P}_{t}^{*}\right\} \\
& \sum_{k=0}^{\infty}(\theta \beta)^{k} E_{t}\left\{\hat{P}_{t}^{*}\right\}=\sum_{k=0}^{\infty}(\theta \beta)^{k} E_{t}\left\{\hat{\psi}_{t+k \mid t}\right\}
\end{aligned}
$$

Sabemos que $\sum_{k=0}^{\infty}(\theta \beta)^{k}=\frac{1}{1-\theta \beta}$, entonces, sustituyendo en la ecuación (A.42):

$$
\hat{P}_{t}^{*}=(1-\theta \beta) \sum_{k=0}^{\infty}(\theta \beta)^{k} E_{t}\left\{\hat{\psi}_{t+k \mid t}\right\}
$$

El costo marginal en términos reales es: $M C_{t+k \mid t}=\frac{\psi_{t+k \mid t}}{P_{t+k}}$ y log-linealizando podemos expresar $\widehat{M C}_{t+k \mid t}=\widehat{\psi}_{t+k \mid t}-\widehat{P}_{t+k}$, entonces reemplazando a la ecuación (A.43):

$$
\hat{P}_{t}^{*}=(1-\theta \beta) \sum_{k=0}^{\infty}(\theta \beta)^{k} E_{t}\left\{\widehat{M C}_{t+k \mid t}+\hat{P}_{t+k}\right\}
$$


Sin el precio $\hat{P}_{t-1}=(1-\theta \beta) \Sigma_{k=0}^{\infty}(\theta \beta)^{k} E_{t}\left\{\hat{P}_{t-1}\right\}$, reemplazando en la ecuación 34:

$$
\hat{P}_{t}^{*}-\hat{P}_{t-1}=(1-\theta \beta) \sum_{k=0}^{\infty}(\theta \beta)^{k} E_{t}\left\{\widehat{M C}_{t+k \mid t}+\hat{P}_{t+k}-\hat{P}_{t-1}\right\}
$$

Ahora eliminamos el costo marginal, entonces, asumimos que el costo marginal log-linealizado es:

$$
\widehat{M C}_{t+k \mid t}=\widehat{M C}_{t+k}-\frac{\alpha \epsilon}{1-a}\left(\hat{P}_{t}^{*}-\hat{P}_{t+k}\right)
$$

Reemplazando (A.46) en (A.45):

$$
\hat{P}_{t}^{*}-\hat{P}_{t-1}=(1-\theta \beta) \sum_{k=0}^{\infty}(\theta \beta)^{k} E_{t}\left\{\widehat{M C}_{t+k}-\frac{\alpha \epsilon}{1-a}\left(\hat{P}_{t}^{*}-\hat{P}_{t+k}\right)+\hat{P}_{t+k}-\hat{P}_{t-1}\right\}
$$

Ahora sumando y restando por $\hat{P}_{t-1}$ :

$$
\hat{P}_{t}^{*}-\hat{P}_{t-1}=(1-\theta \beta) \sum_{k=0}^{\infty}(\theta \beta)^{k} E_{t}\left\{\widehat{M C}_{t+k}-\frac{\alpha \epsilon}{1-a}\left(\hat{P}_{t}^{*}-\hat{P}_{t-1}\right)+\frac{\alpha \epsilon}{1-a}\left(\hat{P}_{t+k}-\hat{P}_{t-1}\right)+\hat{P}_{t+k}-\hat{P}_{t-1}\right\}
$$

Realizando operaciones algebraicas llegamos a la siguiente ecuación: ${ }^{26}$

$$
\hat{P}_{t}^{*}-\widehat{P}_{t-1}=(1-\theta \beta) \Xi \widehat{M C}_{t}+\pi_{t}+\theta \beta E_{t}\left(\hat{P}_{t+1}^{*}-\hat{P}_{t}\right)
$$

Por precio a la calvo: $\hat{P}_{t}^{*}-\hat{P}_{t-1}=\frac{1}{1-\theta}\left(\hat{P}_{t}-\hat{P}_{t-1}\right)$ y reemplazando en la ecuación 36:

$$
\begin{aligned}
& \frac{1}{1-\theta} \pi_{t}=(1-\theta \beta) \Xi \widehat{M C}_{t}+\pi_{t}+\theta \beta E_{t}\left\{\pi_{t+1}\right\} \\
& \pi_{t}=\frac{(1-\theta)(1-\theta \beta) \Xi}{\theta} \widehat{M C}+\beta E_{t}\left\{\pi_{t+1}\right\}
\end{aligned}
$$

También podemos escribir como:

$$
\pi_{t}=k \widehat{M C}_{t}+\beta E_{t}\left\{\pi_{t+1}\right\}
$$

\footnotetext{
${ }^{26}$ Donde $\Xi=\frac{1-\alpha+\alpha \epsilon}{1-\alpha}$.
} 
Anexo B: Función de pérdida social

Función Objetivo de pérdida social:

$$
-\frac{1}{2}\left\{\left[\alpha x_{t}^{2}+\pi_{t}^{2}\right]\right\}+F_{t}
$$

s.a.

$$
\pi_{t}=\lambda x_{t}+f_{t}
$$

La ecuación general:

$$
\max _{x_{t}}-\frac{1}{2}\left\{\left[\alpha x_{t}^{2}+\pi_{t}^{2}\right]+\phi\left[\pi_{t}-\lambda x_{t}\right]\right\}
$$

Aplicando Condición de Primer Orden:

Tabla B. 1: Resultados de la CPO

$\frac{\partial}{\partial x_{t}}:-\alpha x_{t}-\frac{\lambda}{2} \phi=0$
$\frac{\partial}{\partial \pi_{t}}:-\pi_{t}+\frac{1}{2} \phi=0$

Condición optima

$$
x_{t}=-\frac{\lambda}{\alpha} \pi_{t}
$$


EVALUACIÓN DE REGLAS DE TASAS DE INTERÉS DE

POLÍTICAS MONETARIA: UN

ENFOOUE DE EQUILIBRIO

GENERAL ESTOCÁSTICO DINÁMICO

(DGSE)

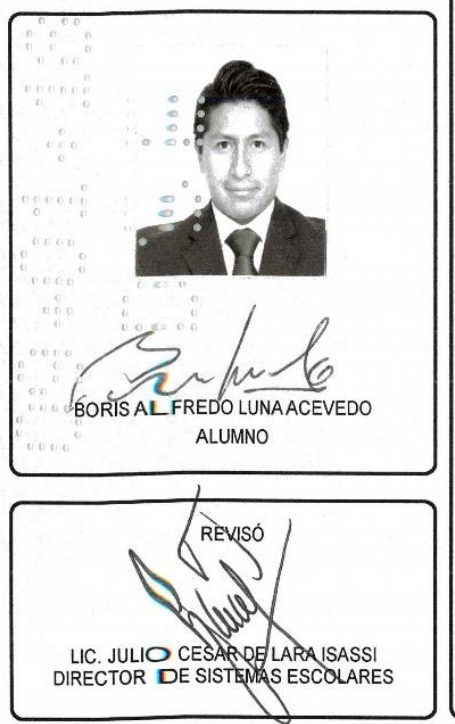

Bajo la Presidencia del primero y con carácter de Secretario el último, se reunieron para proceder al Examen de Grado cuya denominación aparece al margen, para la obtención del grado de:

MAESTRO EN CIENCIAS ECONOMICAS

DE: BORIS ALFREDO LUNA ACEVEDO

y de acuerdo con el artículo 78 fracción III del Reglamento de Estudios Superiores de la Universidad Autónoma Metropolitana, los miembros del jurado resolvieron:

\section{Aprobor}

Acto continuo, el presidente del jurado comunicó al interesado el resultado de la evaluación $y$, en caso aprobatorio, le fue tomada la protesta.
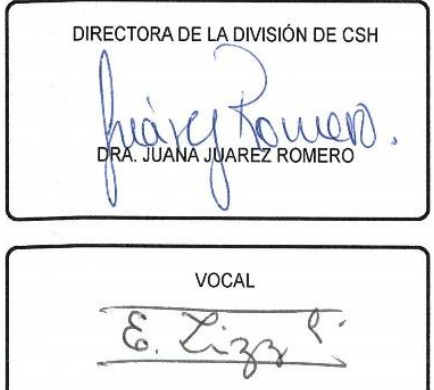

DR. EDDY LIZARAZU ALANEZ
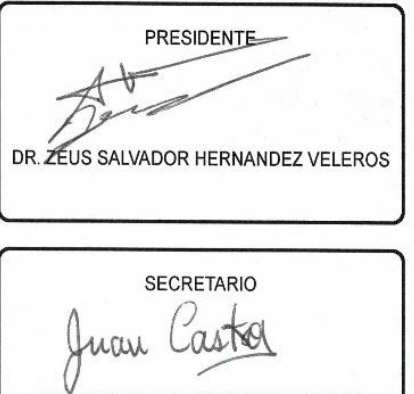

DR. JUAN CARLOS CASTRO RAMIREZ 
A91

UNIVERSIDAD AUTÓNOMA METROPOLITANA

Unidad Iztapalapa

$\begin{array}{lr}\text { Fecha : } & 02 / 01 / 2018 \\ \text { Página : } & 1 / 1\end{array}$

CONSTANCIA DE PRESENTACION DE EXAMEN DE GRADO

La Universidad Autónoma Metropolitana extiende la presente CONSTANCIA DE PRESENTACION DE EXAMEN DE GRADO de MAESTRO EN CIENCIAS ECONOMICAS del alumno BORIS ALFREDO LUNA ACEVEDO, matrícula 2161801834, quien cumplió con los 180 créditos correspondientes a las unidades de enseñanza aprendizaje del plan de estudio. Con fecha tres de enero del 2018 presentó la DEFENSA de su EXAMEN DE GRADO cuya denominación es:

EVALUACIÓN DE REGLAS DE TASAS DE INTERÉS DE POLÍTICAS MONETARIA: UN ENFOOUE DE EQUILIBRIO GENERAL ESTOCÁSTICO DINÁMICO (DGSE)

Cabe mencionar que la aprobación tiene un valor de 40 créditos y el programa consta de 220 créditos.

El jurado del examen ha tenido a bien otorgarle la calificación de:

Aprobado

JURADO
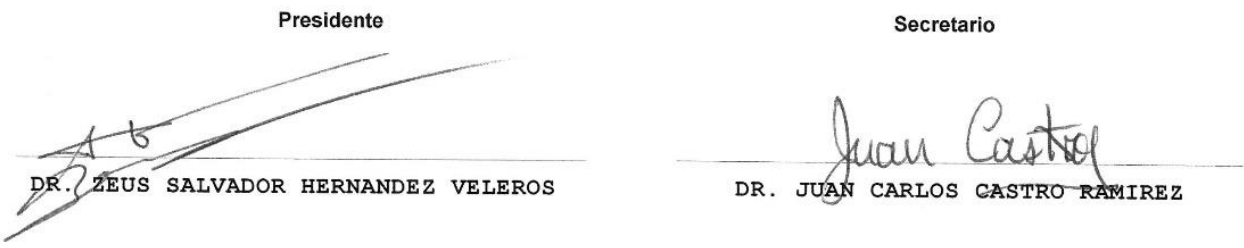

DR. JUAN CARLOS CASTRO RAMIREZ

Vocal

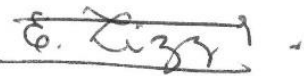

DR. EDDY LIZARAZU ALANEZ 